GLOBAL WATER PATHOGEN PROJECT

PART THREE. SPECIFIC EXCRETED PATHOGENS: ENVIRONMENTAL AND EPIDEMIOLOGY ASPECTS

\title{
NOROVIRUS AND OTHER CALICIVIRUSES
}

\section{Hiroyuki Katayama}

The University of Tokyo

Tokyo, Japan

\section{Jan Vinjé}

Centers for Disease Control and Prevention

Atlanta, United States 


\section{Copyright:}

\section{cc) (i) (2) \\ BY SA}

This publication is available in Open Access under the Attribution-ShareAlike 3.0 IGO (CC-BY-SA 3.0 IGO) license (http://creativecommons.org/licenses/by-sa/3.0/igo). By using the content of this publication, the users accept to be bound by the terms of use of the UNESCO Open Access Repository (http://www.unesco.org/openaccess/terms-use-ccbysa-en).

\section{Disclaimer:}

The designations employed and the presentation of material throughout this publication do not imply the expression of any opinion whatsoever on the part of UNESCO concerning the legal status of any country, territory, city or area or of its authorities, or concerning the delimitation of its frontiers or boundaries. The ideas and opinions expressed in this publication are those of the authors; they are not necessarily those of UNESCO and do not commit the Organization.

\section{Citation:}

Katayama, H. and Vinjé, J. (2017). Norovirus and other Calicivirus. In: J.B. Rose and B. Jiménez-Cisneros, (eds) Water and Sanitation for the 21st Century: Health and Microbiological Aspects of Excreta and Wastewater Management (Global Water Pathogen Project). (J.S Meschke, and R. Girones (eds), Part 3: Specific Excreted Pathogens: Environmental and Epidemiology Aspects - Section 1: Viruses),

Michigan State University, E. Lansing, MI, UNESCO. https://doi.org/10.14321/waterpathogens.14

Acknowledgements: K.R.L. Young, Project Design editor; Website Design: Agroknow (http://www.agroknow.com)

Last published: September 7, 2017 


\section{Summary}

Norovirus is ubiquitous, associated with $18 \%$ (95\% CI: $17-20 \%$ ) of acute gastroenteritis (AGE) worldwide, with similar proportions of disease in high-, middle-, and lowincome settings. Norovirus is estimated to cause approximately 200,000 deaths annually worldwide, with about 70,000-100,000 among children in developing countries. In both high- and middle-income countries with mature rotavirus vaccination programs, norovirus is now also the most common cause of pediatric gastroenteritis requiring medical care. Norovirus is extremely contagious and humans are the only known reservoir for human norovirus. Transmission occurs via direct person-to-person, foodborne, waterborne or through environmental fomites.

Noroviruses are a group of non-enveloped, single-stranded RNA viruses with an icosahedral symmetry classified into the genus Norovirus of the family Caliciviridae. Other genera within this virus family include Sapovirus, which also causes AGE in humans. Infections in humans are caused by viruses of genogroup (G) I, II and IV for norovirus and by GI, II, IV and V for sapoviruses. Since human norovirus and sapovirus cannot be routinely grown in cell culture, detection of the virus relies on sensitive molecular methods such as real-time reverse transcription polymerase chain reaction. In the environment, norovirus can be found in any water that comes into contact with human stool samples which can also lead to contaminated crops (irrigation) and shellfish (growing waters).

Norovirus is highly resistant to environmental degradation in various water types and long-term infectivity has been reported for groundwater which when seeded with the prototype norovirus (GI.1 Norwalk virus) was infectious for at least 61 days. Norovirus contamination of drinking water can be controlled by adequate free chlorine disinfection practices with provision of proper pre-treatment processes before chlorination. Appropriate hand hygiene is likely the single most important method to prevent norovirus infection which is best accomplished through handwashing with water and soap. The use of chemical disinfectants such as sodium hypochlorite is one of the key approaches to disinfect norovirus from contaminated surfaces and drinking water. However, the effectiveness of most water and waste water treatments and disinfection methods on reduction of infectious norovirus can only be measured by the use of cultivable surrogate viruses such as murine norovirus and Tulane virus. An exciting new development is a vaccine against norovirus which is soon entering phase 3 clinical trials.

\subsection{Epidemiology of the Disease and Pathogen(s)}

\subsection{Global Burden of Disease}

\subsubsection{Global distribution}

Although child deaths have declined in recent decades, but diarrheal disease remains the fourth most common cause of mortality and second most common cause of morbidity worldwide in children under the age of 5 years (Global Health Data Exchange. 2013; Available from: http://vizhub.healthdata.org/irank/arrow.php.). Based on a large systematic literature review of 175 studies, which describe over 185,000 cases of acute gastroenteritis, norovirus is associated with $18 \%$ (95\% CI: 17-20\%) of all diarrheal disease worldwide (Ahmed et al., 2014). This percentage is higher among community cases (24\%) than among outpatients (20\%) or inpatients (17\%), which is in line with the notion that norovirus is causing more often less severe clinical symptoms, albeit still an important cause of severe diarrheal disease. As a fraction of all diarrheal diseases, norovirus is more frequently detected in developed countries (20\%) and low-mortality developing countries $(19 \%)$ than those with high-mortality $(14 \%)$ (Ahmed et al., 2014). This lower prevalence in low-income settings likely indicates a more prominent role for other pathogens that are largely controlled through water and sanitation improvements in developed countries, not that norovirus incidence is lower in developing country settings. In the United States, noroviruses are estimated to be associated with 570-800 deaths, 56,000-71,000 hospitalizations and 400,000 emergency department visits annually (Hall et al., 2013). Global age-stratified estimates of illnesses and deaths ranks norovirus as the number 1 cause of foodborne illness and number 4 cause of foodborne deaths (Kirk et al., 2015). The global estimated cost of norovirus disease which includes both direct health system costs and productivity losses is 60 billion per year (Bartsch et al., 2016).

In children under the age of 5 years, an estimated 71,000 die as a result of norovirus gastroenteritis making it the third most common etiological cause of diarrheal mortality, after rotavirus and, possibly, enteropathogenic Escherichia coli (Lanata et al., 2013). However, for norovirus, which causes mild disease in the majority of cases, much of the burden is morbidity, not mortality. The sapoviruses, which initially were thought to only cause AGE in children, are now recognized to cause outbreaks in people of all age groups including the elderly (Lee et al., 2012).

\subsubsection{Symptomology}

Norovirus infection affects individuals of all ages, with the highest rates among young children (Ahmed et al., 2014). With a life expectancy of 80 years, this means that a person will experience an average of approximately three to eight norovirus illness episodes in their lifetime, of which at least one will occur by 5 years of age (Phillips et al., 2010). Noroviruses are the leading cause of outbreaks of acute gastroenteritis and in several countries that have introduced rotavirus vaccines, where they have become also the most frequent cause of sporadic gastroenteritis (Payne et al., 2013).

Norovirus as well as sapovirus gastroenteritis is characterized by acute onset, nonbloody diarrhea, (projectile) vomiting, nausea, and abdominal cramps. Some 
persons might experience only vomiting or diarrhea. Lowgrade fever and body aches also might be associated with infection, and thus the term "stomach flu" often is used to describe the illness but there is no biological association with influenza. Although symptoms might be severe, they typically resolve without treatment after 1-3 days in otherwise healthy persons. However, more prolonged courses of illness lasting 4-6 days can occur, particularly among young children, elderly persons, and hospitalized patients. Worldwide, the WHO estimated that noroviruses annually cause 685 million cases of diarrhea $(95 \%$ confidence interval [CI]: 491 million-1.1 billion) and $212,489$ deaths (95\% CI: $160,595-278,420)$, with 85\% of these illnesses and $\sim 99 \%$ of the deaths occurring in developing countries (Pires et al., 2015)

\subsection{Taxonomic classification of the agent(s)}

\subsubsection{Physical description of the agent}

Norovirus and sapovirus are non-enveloped 30-35 nm particles with an icosahedral symmetry that enclose a positive-sense single-stranded RNA genome of 7.1-7.7 kilobases in length. Most norovirus genomes contain 3 open reading frames (ORF) while the genome organization of sapoviruses differs and contains 2 ORFs. The ORF1 of noroviruses encodes a polyprotein that is posttranslationally cleaved into seven non-structural mature proteins (NS1-7) that are involved in viral replication. ORF2 encodes the major structural protein (VP1) of approximately $60,000 \mathrm{D}$, and ORF3 encodes a minor structural protein (VP2). The ORF1 of sapoviruses encodes the non-structural proteins followed by the major capsid protein in the same reading frame. Viral capsids contains 90 dimers of VP1 and a few gene copies of VP2. X-ray crystallographic structure studies using Norwalk virus-like particles have revealed that the VP1 has a shell (S) and the protruding (P) domain (Prasad et al., 1999). The S domain surrounds the viral RNA and the P domain, which is linked to the S domain through a flexible hinge, corresponds to the C-terminal part of the VP1. The P domain is further divided into the P1 and the highly variable P2 subdomain which contains the putative neutralization sites and interacts with histoblood group antigens (HBGAs). VP2 is located interior to the virus particle and is most likely involved in virion stability (Lin et al., 2014).

\subsubsection{Taxonomy}

Previously, noroviruses were called Norwalk-like viruses or small-round structured viruses whereas sapoviruses were called Sapporo-like viruses or typical caliciviruses. In 2002, the International Committee on the Taxonomy of Viruses assigned Norwalk-like viruses to the species Norwalk virus, genus Norovirus and Sapporo-like viruses to the species Sapporo virus, genus Sapovirus in the family Caliciviridae (Mayo, 2002). Currently, the family Caliciviridae consists of five established genera, including Sapovirus, Norovirus, Lagovirus, Vesivirus, and Nebovirus (http://www.ictvonline.org/virusTaxonomy.asp), whereas five potentially new genera (Bavovirus, Nacovirus, Recovirus, and Valovirus) have been proposed.

Except for murine norovirus (GV) strains and the Cowden-1 porcine sapovirus (GIII) strain, norovirus and sapovirus cannot be routinely cultivated in vitro which prevents the classification of these viruses into distinct serotypes using neutralization. Therefore, classification is based on sequence-based typing into genogroups and to date at least 7 norovirus genogroups (GI-GVII) (Vinjé, 2015) and 7 sapovirus genogroups(Oka et al., 2015) are recognized. Viruses belonging to norovirus GI, GII and GIV infect humans where sapovirus GI, GII, GIV and GV have been reported to cause disease in humans. Each genogroup is further divided into genotypes: 9, 22, and 1 for norovirus GI, GII, and GIV and 7, 7, 1 and 2 for sapovirus GI, GII, GIV and GV, respectively.

Despite the extensive genetic diversity among noroviruses, viruses from a single genotype, GII.4, are responsible for the majority of the norovirus outbreaks worldwide (Siebenga et al., 2009). Since the mid-1990s novel pandemic norovirus GII.4 variants have emerged every 2-3 years replacing previous predominant GII.4 strains. This process is likely driven by evasion of immunity in the human population. These global GII.4 variant strains include the GII.4 US95/96 strain in 1995, GII.4 Farmington Hills in 2002, GII.4 Hunter in 2004, GII.4 Den Haag in 2006, GII.4 New Orleans in 2009 and GII.4 Sydney in 2012. These new GII.4 variants are often, but not always, associated with an increase in the number of outbreaks (Leshem et al., 2013). In 2007, GIV sapoviruses were reported in several European countries suggesting a similar epidemic spread of a single genotype.

\subsection{Transmission}

Norovirus is extremely contagious and although several animal species have been described infected with norovirus, these viruses are genetically and antigenically diverse from the viruses that infect humans. Transmission of both norovirus and sapovirus occurs via fecal-oral and vomit-oral pathways by four general routes including direct person-to-person, foodborne, waterborne or through environmental fomites. The illness typically begins after an incubation period of 12-48 hours. Norovirus is shed primarily in the stool but also can be found in the vomitus of infected persons. Peak viral shedding occurs 2-5 days after infection, with a viral load of approximately 100 billion viral gene copies per gram of feces (Atmar, 2008). The level of noroviruses in the human feces in various settings are summarized in Table 1. 
Table.1 Norovirus concentrations in feces

\begin{tabular}{|c|c|c|c|c|c|c|c|}
\hline Area & Sample Type & Virus Genotype & $\begin{array}{l}\text { Percent } \\
\text { Positive } \\
\text { (\# of } \\
\text { samples) }\end{array}$ & $\begin{array}{l}\text { Median } \\
(G C / L)^{a}\end{array}$ & $\begin{array}{c}\text { Minimum } \\
\text { Concentration } \\
(\mathrm{GC} / \mathrm{L})\end{array}$ & $\begin{array}{c}\text { Maximum } \\
\text { Concentration } \\
(G C / L)\end{array}$ & Reference \\
\hline $\begin{array}{l}\text { Hong } \\
\text { Kong } \\
\text { (China) }\end{array}$ & AGI patients & Norovirus GI & $\begin{array}{c}2.6 \% \\
(17 / 651)\end{array}$ & $8.40 \mathrm{E}+05$ & $2.20 \mathrm{E}+04$ & $3.00 \mathrm{E}+08$ & $\begin{array}{l}\text { Chan et } \\
\text { al., } 2006\end{array}$ \\
\hline $\begin{array}{l}\text { Hong } \\
\text { Kong } \\
\text { (China) }\end{array}$ & AGI patients & Norovirus GII & $\begin{array}{c}7.1 \% \\
(46 / 651)\end{array}$ & $3.00 \mathrm{E}+08$ & $2.50 \mathrm{E}+04$ & $7.70 \mathrm{E}+10$ & $\begin{array}{l}\text { Chan et } \\
\text { al., } 2006\end{array}$ \\
\hline $\begin{array}{l}\text { Hong } \\
\text { Kong } \\
\text { (China) }\end{array}$ & $\begin{array}{l}\text { AGI short } \\
\text { patients }\end{array}$ & Norovirus GII & $\begin{array}{c}62.2 \% \\
(25 / 40)\end{array}$ & $2.40 \mathrm{E}+08$ & $7.76 \mathrm{E}+07$ & $2.82 \mathrm{E}+09$ & $\begin{array}{l}\text { Lee et al., } \\
2007\end{array}$ \\
\hline $\begin{array}{l}\text { Hong } \\
\text { Kong } \\
\text { (China) }\end{array}$ & $\begin{array}{l}\text { AGI long } \\
\text { patients }\end{array}$ & Norovirus GII & $\begin{array}{c}37.8 \% \\
(15 / 40)\end{array}$ & $3.09 \mathrm{E}+10$ & $6.92 \mathrm{E}+08$ & $8.71 \mathrm{E}+10$ & $\begin{array}{l}\text { Lee et al., } \\
2007\end{array}$ \\
\hline Italy & $\begin{array}{l}\text { AGI Infant } \\
\text { patients }\end{array}$ & Norovirus GII & $\begin{array}{l}100 \% \\
(3 / 3)\end{array}$ & NR & $6.10 \mathrm{E}+03$ & $1.40 \mathrm{E}+08$ & $\begin{array}{l}\text { La Rosa et } \\
\text { al., } 2010\end{array}$ \\
\hline Japan & $\begin{array}{l}\text { Feces from } \\
\text { Health } \\
\text { infants }\end{array}$ & Norovirus GII & $\begin{array}{c}12.7 \% \\
(17 / 134)\end{array}$ & NR & NR & NR & $\begin{array}{l}\text { Iwai et al., } \\
2009\end{array}$ \\
\hline USA & $\begin{array}{l}\text { Traveler ( } \\
>18) \text { AGI } \\
\text { patients }\end{array}$ & Norovirus GI & $\begin{array}{c}3.8 \% \\
(12 / 320)\end{array}$ & $1.73 \mathrm{E}+10$ & $3.76 \mathrm{E}+07$ & $1.18 \mathrm{E}+13$ & $\begin{array}{l}\text { Ajami et } \\
\text { al., } 2010\end{array}$ \\
\hline USA & $\begin{array}{l}\text { Traveler ( } \\
\text { >18) AGI } \\
\text { patients }\end{array}$ & Norovirus GII & $\begin{array}{c}5.6 \% \\
(18 / 320)\end{array}$ & $3.43 \mathrm{E}+07$ & $3.26 \mathrm{E}+05$ & $8.93 E+09$ & $\begin{array}{l}\text { Ajami et } \\
\text { al., } 2010\end{array}$ \\
\hline USA & $\begin{array}{l}\text { Inoculum for } \\
\text { Volunteer }\end{array}$ & Norovirus GI & $\begin{array}{c}100 \% \\
(1 / 1)\end{array}$ & $3.24 \mathrm{E}+08$ & NR & NR & $\begin{array}{l}\text { Teunis et } \\
\text { al., } 2008\end{array}$ \\
\hline USA & $\begin{array}{c}\text { Volunteer } \\
\text { symptomatic }\end{array}$ & Norovirus GI & $\begin{array}{c}69 \% \\
(11 / 16)\end{array}$ & $2.50 \mathrm{E}+11$ & $3.16 \mathrm{E}+09$ & $1.58 \mathrm{E}+12$ & $\begin{array}{l}\text { Atmar et } \\
\text { al., } 2008\end{array}$ \\
\hline USA & $\begin{array}{c}\text { Volunteer } \\
\text { asymptomatic }\end{array}$ & Norovirus GI & $\begin{array}{c}31 \% \\
(5 / 16)\end{array}$ & $1.20 \mathrm{E}+10$ & $1.58 \mathrm{E}+09$ & $5.01 \mathrm{E}+11$ & $\begin{array}{l}\text { Atmar et } \\
\text { al., } 2008\end{array}$ \\
\hline
\end{tabular}

NR: Not Reported; ${ }^{a}$ Method was Real-time PCR GC=gene copy calculated from copy/gram

Although norovirus is highly contagious, not all individuals are susceptible to infection. This is dependent on the expression of histo blood group antigens (HBGAs) on gut epithelial surfaces where the virus is believed to attach to the human host (Marionneau et al., 2002). Persons carrying a functional fucosyltransferase 2 (encoded by FUT2 gene) are termed secretors and express HBGAs, whereas homozygous individuals with $428 \mathrm{G}>\mathrm{A}$ nonsense mutations in the FUT2 gene, individual called nonsecretors, are almost completely protected from GI.1 and GII.4 norovirus infections. However, polymorphisms in the FUT2 genes vary considerably depending on ethnicity and non-secretors can be infected by other norovirus genotypes (Jin et al., 2013).

\subsection{Population and Individual Control Measures}

\subsubsection{Vaccines}

Since human noroviruses do not replicate in cell culture, recombinant norovirus capsid proteins which can be expressed as virus like particles (VLPs), have been considered as potential antigens for norovirus vaccines. VLPs are morphologically and antigenically indistinguishable to native viruses, but lack genetic material, so are non-replicating. Early studies showed that VLPs can elicit a humoral and mucosal response in mice and humans by oral, intranasal or parenteral administration (Herbst-Kralovetz et al., 2010; Tacket et al., 2003). A number of norovirus vaccines are now under development, and all are based on expressed VLPs.

Noroviruses are genetically and antigenically diverse. Acquired immunity is of limited duration against homotypic strains, there is some degree of protection against viruses in the same genogroup, but there is little or no heterotypic protection across genogroups. Accordingly, a norovirus vaccine would likely need to be at least bivalent to protect against GI and GII viruses. So far, all vaccine trials have involved challenge with a genotype included in the vaccine. Recently, Lindesmith and colleagues analyzed sera from human volunteers who had been immunized with a bivalent norovirus vaccine containing GI.1 and GII.4 virus-like particles (VLPs)(Lindesmith et al., 2015) of which the GII.4 component is a consensus (GII.4C) based on major capsid 
protein sequences from three GII.4 variants (GII.4 Houston/2002, GII.4 Yerseke/2006a and GII.4 DenHaag/2006b). At day 7-post vaccination substantial rises of IgG and blockade Abs were observed against GI.1 and GII.4 and also against GI.3, GII.3 and GII.14 VLPs, suggesting broad and rapid antibody response. That GI.1 and GII.4 VLPs would elicit a response to other genotypes in humans was hardly a foregone conclusion given the extreme antigenic diversity of noroviruses. This observation is consistent with observations of humans infected during experimental GI.1 Norwalk virus studies who mounted not only histoblood group blocking antibody responses to homotypic GI.1 VLPs but also responses to other norovirus VLPs including GII.4 Sydney that had not yet circulated at the time of the infections (Czakó et al., 2015; Lindesmith et al., 2010).

The norovirus vaccine candidate furthest along in the development pipeline is a bivalent, intramuscular VLP vaccine which is being developed by Takeda Pharmaceuticals (Bernstein et al., 2015). This formulation has shown a degree of protection against severe gastroenteritis outcomes following GII.4 challenge. Efficacy clinical trials using the bivalent GI/GII.4 formulation are planned to start in military recruits, followed by trials in the elderly and infants. One of the challenges in developing norovirus vaccines is that so many distinct (or different) population subgroups are affected which complicates the formulation of a research agenda and clinical development plan. For example, vaccination protocols would be different for a target population of young children, who likely would require at least two shots, than it would for older adults for which one booster shot may be sufficient for protection, or for a specific risk group, such as healthcare workers.

\subsubsection{Hygiene measures}

The main approaches to preventing and containing norovirus infections include implementing policies surrounding hand hygiene, patient isolation (separation of symptomatic patients) and cohorting (grouping of patients based on symptoms), staff exclusion from work, visitor restrictions, enhanced environmental cleaning and disinfection, and ward closures (Barclay et al., 2014). In general, hand hygiene adherence should be actively promoted among healthcare personnel, patients and visitors in patient-care areas affected by outbreaks of norovirus gastroenteritis. During outbreaks, hands should be washed with soap and running water for a minimum of $20 \mathrm{~s}$ after providing care for patients with suspected or confirmed infection (MacCannell et al., 2011; CDC et al., 2011). Despite widespread use, there is inconclusive evidence for the effectiveness of alcohol-based hand sanitizers for norovirus (Park et al., 2010; Macinga et al., 2008; Sickbert-Bennett et al., 2005). Therefore, during outbreaks, they should be used as an adjunct to handwashing (Hall et al., 2011). Since aerosolization of noroviruses and close, direct contact with an infected individual contribute to the high risk of transmission (Phillips, 2011), the use of appropriate personal protective equipment, i.e., gloves and masks, when cleaning up vomit, is another measure to limit further spread of norovirus infection to staff in healthcare facilities (MacCannell et al., 2011).

\subsection{Environmental Occurrence, Persistence and Survival}

\subsection{Detection methods}

Because no robust cell culture system for norovirus or sapovirus currently exist, methods to detect these viruses are based on detection of viral RNA primarily using polymerase chain reaction (PCR). Since the initial description of PCR (Saiki et al., 1985), reverse transcription (RT)-PCR and, more recently, real-time RT-PCR (RT-qPCR) methods have become the gold standard for detection of norovirus in both clinical and environmental samples. Various combinations of oligonucleotide primers and probes for the sensitive detection of norovirus in food and water have been reported (Kageyama et al., 2003; Stals et al., 2012; Vinjé, 2015). Standardized method have been developed for detecting both of these viruses (TAG 4 of CEN/TC 275/WG 6) and have been approved (CEN ISO/TS 15216-parts 1 \& 2)(Hennechart-Collette et al., 2015; Standardization, 2013a, 2013b).

\subsection{Data on occurrence}

\subsubsection{Sewage and sludge}

In temperate climates, norovirus and sapovirus occurrence is quite seasonal with most infections during the winter months (Katayama et al., 2008). After development of PCR-based detection methods for norovirus and sapovirus, many papers have been published on the presence of these viruses in sewer systems from different regions of the world. The concentration of enteric viruses in the wastewater influent fluctuates dependent on timing of sampling. The occurrence data are summarized in Table 2. Here, the maximum concentration in the raw sewage reported is focused since it is important from a risk management perspective whereas lower virus concentrations reported may be the result of RT-PCR inhibition and/or low recovery of viruses during concentration. Maximum concentration of norovirus GI ranged from $10^{5.4}$ gene copies /L (Haramoto, 2006) to $10^{6.7}$ gene copies /L (Katayama, 2008) with one paper reporting even $10^{9}$ gene copies /L (Da Silva, 2007). Maximum concentration of norovirus GII ranged from $10^{5.6}$ gene copies /L (Kitajima, 2014) to $10^{8.6}$ gene copies /L (Simmons, 2011). Concentration of sapovirus (GI, GII, GIV and GV) ranged generally from $10^{3}$ to $10^{4}$ (Kitajima, 2011), but is more fluctuated, some reporting $10^{8,3}$ gene copies /L (Sano, 2011). In general, the concentration of sapoviruses is less than noroviruses (Iwai, 2009). 
Table. 2 Calicivirus concentrations in raw sewage

\begin{tabular}{|c|c|c|c|c|}
\hline Area & $\begin{array}{c}\text { Virus } \\
\text { Genotype }\end{array}$ & $\begin{array}{l}\text { Percent Positive } \\
\text { (\# of samples) }\end{array}$ & $\begin{array}{c}\text { Concentrations } \\
\text { Average } \\
\text { GC/L } \mathbf{L}^{\mathrm{a}} \\
\text { (Range) } \\
\end{array}$ & Reference \\
\hline France & $\begin{array}{l}\text { Norovirus } \\
\text { GI }\end{array}$ & $\begin{array}{c}43 \% \\
(34 / 79)\end{array}$ & $(1.00 \mathrm{E}+09 \mathrm{Max})$ & $\begin{array}{l}\text { da Silva et } \\
\text { al., } 2007\end{array}$ \\
\hline France & $\begin{array}{l}\text { Norovirus } \\
\text { GII }\end{array}$ & $\begin{array}{c}88 \% \\
(71 / 81)\end{array}$ & $(6.00 \mathrm{E}+07 \mathrm{Max})$ & $\begin{array}{c}\text { da Silva et } \\
\text { al., } 2007\end{array}$ \\
\hline Ireland & $\begin{array}{l}\text { Norovirus } \\
\text { GII }\end{array}$ & $\begin{array}{c}95 \% \\
(21 / 22)\end{array}$ & $7.40 \mathrm{E}+04$ & $\begin{array}{c}\text { Flannery et } \\
\text { al., } 2013\end{array}$ \\
\hline Italy & $\begin{array}{l}\text { Noroviruses } \\
\text { GI }\end{array}$ & $\begin{array}{l}100 \% \\
(6 / 6)\end{array}$ & $\begin{array}{c}(6.46 \mathrm{E}+05 \text { to } \\
5.73 \mathrm{E}+07)\end{array}$ & $\begin{array}{c}\text { La Rosa et } \\
\text { al., } 2010\end{array}$ \\
\hline Italy & $\begin{array}{c}\text { Noroviruses } \\
\text { GII }\end{array}$ & $\begin{array}{c}100 \% \\
(12 / 12)\end{array}$ & $\begin{array}{c}(6.80 \mathrm{E}+05 \text { to } \\
6.77 \mathrm{E}+07)\end{array}$ & $\begin{array}{l}\text { La Rosa et } \\
\text { al., } 2010\end{array}$ \\
\hline Italy & Norovirus & $\begin{array}{c}72 \% \\
(17 / 23) \\
\text { in both rainy and dry }\end{array}$ & $\begin{array}{c}6.76 \mathrm{E}+05 \\
\left(\mathrm{SD}^{\mathrm{b}} \pm 7.41 \mathrm{E}+02\right)^{\mathrm{c}}\end{array}$ & $\begin{array}{c}\text { Carducci } \\
\text { and Verani, } \\
2013\end{array}$ \\
\hline Italy & Norovirus & $\begin{array}{c}72 \% \\
(17 / 23) \\
\text { in both rainy and dry }\end{array}$ & $\begin{array}{c}8.32 \mathrm{E}+05^{\mathrm{d}} \\
(\mathrm{SD} \pm 7.24 \mathrm{E}+02)\end{array}$ & $\begin{array}{c}\text { Carducci } \\
\text { and Verani, } \\
2013\end{array}$ \\
\hline Japan & $\begin{array}{l}\text { Norovirus } \\
\text { GI }\end{array}$ & $\begin{array}{c}100 \% \\
(12 / 12)\end{array}$ & $\begin{array}{c}(1.70 \mathrm{E}+02 \text { to } \\
2.60 \mathrm{E}+05\end{array}$ & $\begin{array}{l}\text { Haramoto } \\
\text { et al., } 2006\end{array}$ \\
\hline Japan & $\begin{array}{l}\text { Norovirus } \\
\text { GII }\end{array}$ & $\begin{array}{c}100 \% \\
(12 / 12)\end{array}$ & $\begin{array}{c}(2.40 \mathrm{E}+03 \text { to } \\
1.90 \mathrm{E}+06)\end{array}$ & $\begin{array}{l}\text { Haramoto } \\
\text { et al., } 2006\end{array}$ \\
\hline Japan & Sapovirus & $\begin{array}{c}100 \% \\
(12 / 12)\end{array}$ & $\begin{array}{c}(2.80 \mathrm{E}+03 \text { to } \\
1.30 \mathrm{E}+05)\end{array}$ & $\begin{array}{l}\text { Haramoto } \\
\text { et al., } \\
2008 b\end{array}$ \\
\hline Japan & $\begin{array}{l}\text { Norovirus } \\
\text { GI }\end{array}$ & $\begin{array}{c}96 \% \\
(23 / 24)\end{array}$ & $(2.30 \mathrm{E}+06 \mathrm{Max})$ & $\begin{array}{l}\text { Iwai et al., } \\
2009\end{array}$ \\
\hline Japan & $\begin{array}{l}\text { Norovirus } \\
\text { GII }\end{array}$ & $\begin{array}{l}100 \% \\
(24 / 24)\end{array}$ & $(7.10 \mathrm{E}+07 \mathrm{Max})$ & $\begin{array}{l}\text { Iwai et al., } \\
2009\end{array}$ \\
\hline Japan & $\begin{array}{l}\text { Norovirus } \\
\text { GI }\end{array}$ & $\begin{array}{l}100 \% \\
(24 / 24)\end{array}$ & $\begin{array}{l}4.90 \mathrm{E}+03^{\mathrm{e}} \\
(\mathrm{SD} \pm 0.55)\end{array}$ & $\begin{array}{l}\text { Katayama } \\
\text { et al., } 2008\end{array}$ \\
\hline Japan & $\begin{array}{l}\text { Norovirus } \\
\text { GI }\end{array}$ & $\begin{array}{c}100 \% \\
(30 / 30)\end{array}$ & $\begin{array}{c}1.90 \mathrm{E}+05^{\mathrm{f}} \\
(\mathrm{SD} \pm 0.57)\end{array}$ & $\begin{array}{c}\text { Katayama } \\
\text { et al., } 2008\end{array}$ \\
\hline Japan & $\begin{array}{l}\text { Norovirus } \\
\text { GII }\end{array}$ & $\begin{array}{c}100 \% \\
(24 / 24)\end{array}$ & $\begin{array}{c}9.10 \mathrm{E}+03^{\mathrm{e}} \\
(\mathrm{SD} \pm 0.56)\end{array}$ & $\begin{array}{c}\text { Katayama } \\
\text { et al., } 2008\end{array}$ \\
\hline Japan & $\begin{array}{l}\text { Norovirus } \\
\text { GII }\end{array}$ & $\begin{array}{c}100 \% \\
(30 / 30)\end{array}$ & $\begin{array}{c}2.00 \mathrm{E}+05^{\mathrm{f}} \\
(\mathrm{SD} \pm 0.63)\end{array}$ & $\begin{array}{c}\text { Katayama } \\
\text { et al., } 2008\end{array}$ \\
\hline Japan & $\begin{array}{l}\text { Norovirus } \\
\text { GIV }\end{array}$ & $\begin{array}{c}50 \% \\
(6 / 12)\end{array}$ & $(6.90 \mathrm{E}+04 \mathrm{Max})$ & $\begin{array}{l}\text { Kitajima et } \\
\text { al., } 2009\end{array}$ \\
\hline Japan & Sapovirus & $\begin{array}{c}100 \% \\
(12 / 12)\end{array}$ & $\begin{array}{c}1.80 \mathrm{E}+04 \\
(\mathrm{SD} \pm 0.47) \\
(9.30 \mathrm{E}+04 \mathrm{Max})\end{array}$ & $\begin{array}{l}\text { Kitajima et } \\
\text { al., } 2011\end{array}$ \\
\hline Japan & $\begin{array}{l}\text { Norovirus } \\
\text { GI }\end{array}$ & $\begin{array}{c}100 \% \\
(12 / 12)\end{array}$ & $(1.50 \mathrm{E}+06 \mathrm{Max})$ & $\begin{array}{l}\text { Kitajima et } \\
\text { al., } 2012\end{array}$ \\
\hline Japan & $\begin{array}{c}\text { Norovirus } \\
\text { GII }\end{array}$ & $\begin{array}{c}100 \% \\
(12 / 12)\end{array}$ & $(9.10 \mathrm{E}+05 \mathrm{Max})$ & $\begin{array}{l}\text { Kitajima et } \\
\text { al., } 2012\end{array}$ \\
\hline Japan & $\begin{array}{l}\text { Norovirus } \\
\text { GII }\end{array}$ & $\begin{array}{c}95 \% \\
(15 / 16)\end{array}$ & $1.20 \mathrm{E}+06$ & $\begin{array}{l}\text { Miura et } \\
\text { al., } 2015\end{array}$ \\
\hline Japan & Sapovirus & $\begin{array}{c}68 \% \\
(11 / 16)\end{array}$ & $2.70 \mathrm{E}+04$ & $\begin{array}{l}\text { Miura et } \\
\text { al., } 2015\end{array}$ \\
\hline Netherlands & Noroviruses & $\begin{array}{c}100 \% \\
(58 / 58 \square\end{array}$ & $\begin{array}{c}(5.10 \mathrm{E}+03 \text { to } \\
8.50 \mathrm{E}+05)\end{array}$ & $\begin{array}{l}\text { Lodder and } \\
\text { Husman, } \\
2005\end{array}$ \\
\hline $\begin{array}{l}\text { New } \\
\text { Zealand }\end{array}$ & $\begin{array}{c}\text { Norovirus } \\
\text { GI }\end{array}$ & $\begin{array}{c}82 \% \\
(9 / 11)\end{array}$ & $\begin{array}{l}1.26 \mathrm{E}+03 \\
(\mathrm{SD} \pm 1.1)\end{array}$ & $\begin{array}{c}\text { Wolf et al., } \\
2010\end{array}$ \\
\hline
\end{tabular}




\begin{tabular}{|c|c|c|c|c|}
\hline Area & $\begin{array}{c}\text { Virus } \\
\text { Genotype }\end{array}$ & $\begin{array}{l}\text { Percent Positive } \\
\text { (\# of samples) }\end{array}$ & $\begin{array}{c}\text { Concentrations } \\
\text { Average } \\
\text { GC/L }{ }^{\mathrm{a}} \\
\text { (Range) } \\
\end{array}$ & Reference \\
\hline $\begin{array}{l}\text { New } \\
\text { Zealand }\end{array}$ & $\begin{array}{l}\text { Norovirus } \\
\text { GII }\end{array}$ & $\begin{array}{c}82 \% \\
(9 / 11)\end{array}$ & $\begin{array}{l}2.50 \mathrm{E}+04 \\
(\mathrm{SD} \pm 1.0)\end{array}$ & $\begin{array}{l}\text { Wolf et al., } \\
2010\end{array}$ \\
\hline Spain & $\begin{array}{l}\text { Norovirus } \\
\text { GI }\end{array}$ & $\begin{array}{c}91 \% \\
(49 / 54)\end{array}$ & $(5.90 \mathrm{E}+08 \mathrm{Max})$ & $\begin{array}{l}\text { Pérez-Sautu } \\
\text { et al., } 2012\end{array}$ \\
\hline Spain & $\begin{array}{l}\text { Norovirus } \\
\text { GII }\end{array}$ & $\begin{array}{c}98 \% \\
(53 / 54)\end{array}$ & (3.40E+09 Max) & $\begin{array}{l}\text { Pérez-Sautu } \\
\text { et al., } 2012\end{array}$ \\
\hline Spain & Sapovirus & & $(1.80 \mathrm{E}+08 \mathrm{Max})$ & $\begin{array}{c}\text { Sano et al., } \\
2011\end{array}$ \\
\hline Sweden & $\begin{array}{l}\text { Norovirus } \\
\text { GI }\end{array}$ & $\begin{array}{l}100 \% \\
(12 / 12)\end{array}$ & $\begin{array}{c}(1.10 \mathrm{E}+04 \text { to } \\
2.20 \mathrm{E}+06)\end{array}$ & $\begin{array}{l}\text { Nordgren } \\
\text { et al., } 2009\end{array}$ \\
\hline Sweden & $\begin{array}{l}\text { Norovirus } \\
\text { GII }\end{array}$ & $\begin{array}{c}100 \% \\
(12 / 12)\end{array}$ & $(9.40 \mathrm{E}+06 \mathrm{Max})$ & $\begin{array}{c}\text { Nordgren } \\
\text { et al., } 2009\end{array}$ \\
\hline Sweden & Noroviruses & $\begin{array}{c}36 \% \\
(8 / 22)\end{array}$ & $\begin{array}{c}3.00 \mathrm{E}+02 \\
(\mathrm{SD} \pm 0.6) \\
(4.50 \mathrm{E}+03 \mathrm{Max})\end{array}$ & $\begin{array}{l}\text { Ottoson et } \\
\text { al., } 2006\end{array}$ \\
\hline Switzerland & $\begin{array}{l}\text { Norovirus } \\
\text { GII }\end{array}$ & $\begin{array}{c}97 \% \\
(58 / 60)\end{array}$ & $2.68 \mathrm{E}+05$ & $\begin{array}{l}\text { Masclaux, } \\
2013\end{array}$ \\
\hline Uruguay & $\begin{array}{l}\text { Norovirus } \\
\text { GII }\end{array}$ & $\begin{array}{c}51 \% \\
(49 / 96)\end{array}$ & $9.40 \mathrm{E}+05$ & $\begin{array}{l}\text { Victoria, } \\
2014\end{array}$ \\
\hline USA & $\begin{array}{l}\text { Norovirus } \\
\text { GII }\end{array}$ & $\begin{array}{l}100 \% \\
(8 / 8)\end{array}$ & $\begin{array}{c}5.00 \mathrm{E}+07 \\
(1.30 \mathrm{E}+05 \text { to } \\
4.00 \mathrm{E}+08)\end{array}$ & $\begin{array}{l}\text { Simmons et } \\
\text { al., } 2011\end{array}$ \\
\hline USA & $\begin{array}{l}\text { Norovirus } \\
\text { GII }\end{array}$ & $\begin{array}{l}75 \% \\
(6 / 8)\end{array}$ & $3.20 \mathrm{E}+05^{\mathrm{g}}$ & $\begin{array}{l}\text { Simmons et } \\
\text { al., } 2011\end{array}$ \\
\hline
\end{tabular}

${ }^{a}$ GC gene copies; ${ }^{b}$ SD standard deviation ${ }^{\mathrm{c}}$ collected during the rainy season; ${ }^{\mathrm{d}}$ collected duiring dry season $\mathrm{r} ;{ }^{\mathrm{e}}$ collected in summer; ${ }^{\mathrm{f}}$ collected in winter; ${ }^{g}$ post preliminary treatment primary sewage

Detection of enteric viruses in sludge samples can be quite challenging as methods that have been optimized using spiking of viruses into the sludge do not always reflect the fate of indigenous virus originally present. The concentration of norovirus GII in sludge samples in membrane bioreactor were much lower than other enteric viruses that were detected (Simmons et al., 2011). Similar trends was also observed in a report from Japan where sapovirus was more abundant than norovirus in sludge samples (Miura, 2015).

A survey of air samples in a wastewater treatment plant in Denmark showed that norovirus GI was more frequently detected than norovirus GII (Uhrbrand 2011).

\subsubsection{Surface waters}

Surface waters, often receiving treated and in some occasion untreated wastewater, are also containing enteric viruses. Norovirus GI and GII in treated wastewater are usually less than $10^{5}$ gene copies /L (Table 3) (Miura et al., 2015; Katayama et al., 2008), but sometimes reach $10^{6.8}$ gene copies /L (Da Silva et al., 2007) and $10^{6.7}$ gene copies /L(Nordgren et al., 2009). Sapovirus concentration in treated wastewater is not well documented, but reported to reach $10^{3.9}$ gene copies /L (Table 3) (Kitajima et al., 2011). 
Table. 3 Calicivirus concentrations in treated wastewater

\begin{tabular}{|c|c|c|c|c|}
\hline Area & $\begin{array}{l}\text { Virus } \\
\text { Genotype }\end{array}$ & $\begin{array}{l}\text { Percent Positive } \\
\text { (\# of Samples) }\end{array}$ & $\begin{array}{c}\text { Concentrations } \\
\text { Average } \\
\text { GC/L } \mathbf{L}^{\mathbf{a}} \\
\text { (Range) } \\
\end{array}$ & Reference \\
\hline Brazil & $\begin{array}{l}\text { Norovirus } \\
\text { GI }\end{array}$ & NR & $\begin{array}{c}2.97 \mathrm{E}+02 \\
(2.06 \mathrm{E}+02 \text { to } \\
4.29 \mathrm{E}+02)\end{array}$ & $\begin{array}{l}\text { Victoria, } \\
2010\end{array}$ \\
\hline Brazil & $\begin{array}{l}\text { Norovirus } \\
\text { GII }\end{array}$ & NR & $\begin{array}{c}4.40 \mathrm{E}+02 \\
(1.80 \mathrm{E}+02 \text { to } \\
1.09 \mathrm{E}+03)\end{array}$ & $\begin{array}{l}\text { Victoria, } \\
2010\end{array}$ \\
\hline France & $\begin{array}{l}\text { Norovirus } \\
\quad \text { GI }\end{array}$ & $\begin{array}{c}24 \% \\
(18 / 75)\end{array}$ & $(6.00 \mathrm{E}+06 \mathrm{Max})$ & $\begin{array}{l}\text { da Silva et } \\
\text { al., } 2007\end{array}$ \\
\hline France & $\begin{array}{l}\text { Norovirus } \\
\text { GII }\end{array}$ & $\begin{array}{c}14 \% \\
(11 / 71)\end{array}$ & $(3.00 \mathrm{E}+06 \mathrm{Max})$ & $\begin{array}{l}\text { da Silva et } \\
\text { al., } 2007\end{array}$ \\
\hline Ireland & $\begin{array}{l}\text { Norovirus } \\
\text { GII }\end{array}$ & 22 & $2.90 \mathrm{E}+04$ & $\begin{array}{l}\text { Flannery et } \\
\text { al., } 2013\end{array}$ \\
\hline Italy & Norovirus & $\begin{array}{c}65 \% \\
(15 / 23) \text { in both rainy and dry }\end{array}$ & $\begin{array}{c}6.31 \mathrm{E}+05 \\
\left(\mathrm{SD}^{\mathrm{b}}\right. \\
\pm 5.62 \mathrm{E}+02)\end{array}$ & $\begin{array}{c}\text { Carducci } \\
\text { and Verani, } \\
2013\end{array}$ \\
\hline Italy & Norovirus & $\begin{array}{c}65 \% \\
(15 / 23) \text { in both rainy and dry }\end{array}$ & $\begin{array}{c}1.10 \mathrm{E}+06 \\
(\mathrm{SD} \pm 8.71 \mathrm{E}+02)\end{array}$ & $\begin{array}{c}\text { Carducci } \\
\text { and Verani, } \\
2013\end{array}$ \\
\hline Japan & $\begin{array}{l}\text { Norovirus } \\
\text { GI }\end{array}$ & $\begin{array}{l}100 \% \\
(12 / 12)\end{array}$ & $(7.70 \mathrm{E}+02 \mathrm{Max})$ & $\begin{array}{l}\text { Haramoto } \\
\text { et al., } 2006\end{array}$ \\
\hline Japan & $\begin{array}{l}\text { Norovirus } \\
\text { GII }\end{array}$ & $\begin{array}{c}83 \% \\
(10 / 12)\end{array}$ & $(1.50 \mathrm{E}+03 \mathrm{Max})$ & $\begin{array}{l}\text { Haramoto } \\
\text { et al., } 2006\end{array}$ \\
\hline Japan & Sapovirus & $\begin{array}{l}58 \% \\
(7 / 12)\end{array}$ & (5.30E+02 Max) & $\begin{array}{l}\text { Haramoto } \\
\text { et al., } \\
2008 b\end{array}$ \\
\hline Japan & $\begin{array}{l}\text { Norovirus } \\
\text { GI }\end{array}$ & $\begin{array}{c}94 \% \\
(68 / 72)\end{array}$ & $\begin{array}{c}1.40 \mathrm{E}+03 \\
(\mathrm{SD} \pm 7.8) \\
(2.90 \mathrm{E}+04 \mathrm{Max})\end{array}$ & $\begin{array}{l}\text { Katayama } \\
\text { et al., } 2008\end{array}$ \\
\hline Japan & $\begin{array}{l}\text { Norovirus } \\
\text { GII }\end{array}$ & $\begin{array}{c}92 \% \\
(66 / 72)\end{array}$ & $\begin{array}{c}8.10 \mathrm{E}+02 \\
(\mathrm{SD} 6.0) \\
(5.50 \mathrm{E}+04 \mathrm{Max})\end{array}$ & $\begin{array}{l}\text { Katayama } \\
\text { et al., } 2008\end{array}$ \\
\hline Japan & $\begin{array}{l}\text { Norovirus } \\
\text { GIV }\end{array}$ & $\begin{array}{l}25 \% \\
(4 / 12)\end{array}$ & $(4.80 \mathrm{E}+03 \mathrm{Max})$ & $\begin{array}{l}\text { Kitajima et } \\
\text { al., } 2009\end{array}$ \\
\hline Japan & Sapovirus & $\begin{array}{l}42 \% \\
(5 / 12)\end{array}$ & $\begin{array}{c}4.30 \mathrm{E}+03 \\
(\mathrm{SD} \pm 1.6) \\
(8.10 \mathrm{E}+03 \mathrm{Max})\end{array}$ & $\begin{array}{c}\text { Kitajima et } \\
\text { al., } 2011\end{array}$ \\
\hline Japan & $\begin{array}{c}\text { Norovirus } \\
\text { GI }\end{array}$ & $\begin{array}{c}50 \% \\
(6 / 12)\end{array}$ & (5.60E+03 Max) & $\begin{array}{l}\text { Kitajima et } \\
\text { al., } 2012\end{array}$ \\
\hline Japan & $\begin{array}{l}\text { Norovirus } \\
\text { GII }\end{array}$ & $\begin{array}{l}50 \% \\
(6 / 12)\end{array}$ & $(3.80 \mathrm{E}+03 \mathrm{Max})$ & $\begin{array}{c}\text { Kitajima et } \\
\text { al., } 2012\end{array}$ \\
\hline Japan & $\begin{array}{c}\text { Norovirus } \\
\text { GII }\end{array}$ & $\begin{array}{c}26 \% \\
(4 / 16)\end{array}$ & $\begin{array}{c}(4.50 \mathrm{E}+04 \text { to } \\
1.10 \mathrm{E}+05)\end{array}$ & $\begin{array}{l}\text { Miura et } \\
\text { al., } 2015\end{array}$ \\
\hline Japan & Sapovirus & $\begin{array}{c}0 \% \\
(0 / 16)\end{array}$ & NR & $\begin{array}{l}\text { Miura et } \\
\text { al., } 2015\end{array}$ \\
\hline Netherlands & Noroviruses & $\begin{array}{l}100 \% \\
(5 / 5 \square\end{array}$ & $\begin{array}{c}(9.00 \mathrm{E}+02 \text { to } \\
7.50 \mathrm{E}+03)\end{array}$ & $\begin{array}{c}\text { Lodder and } \\
\text { Husman, } \\
2005\end{array}$ \\
\hline Spain & $\begin{array}{l}\text { Norovirus } \\
\text { GI }\end{array}$ & $\begin{array}{c}94 \% \\
(17 / 18)\end{array}$ & NR & $\begin{array}{l}\text { Pérez-Sautu } \\
\text { et al., } 2012\end{array}$ \\
\hline Spain & $\begin{array}{c}\text { Norovirus } \\
\text { GII }\end{array}$ & $\begin{array}{c}100 \% \\
(18 / 18)\end{array}$ & NR & $\begin{array}{l}\text { Pérez-Sautu } \\
\text { et al., } 2012\end{array}$ \\
\hline Sweden & $\begin{array}{l}\text { Norovirus } \\
\text { GI }\end{array}$ & $\begin{array}{c}100 \% \\
(12 / 12)\end{array}$ & (7.90E+05 Max) & $\begin{array}{l}\text { Nordgren } \\
\text { et al., } 2009\end{array}$ \\
\hline
\end{tabular}




\begin{tabular}{|c|c|c|c|c|}
\hline Area & $\begin{array}{c}\text { Virus } \\
\text { Genotype }\end{array}$ & $\begin{array}{l}\text { Percent Positive } \\
\text { (\# of Samples) }\end{array}$ & $\begin{array}{c}\text { Concentrations } \\
\text { Average } \\
\text { GC/L } \mathbf{L}^{\mathrm{a}} \\
\text { (Range) }^{\text {Range }} \\
\end{array}$ & Reference \\
\hline Sweden & $\begin{array}{l}\text { Norovirus } \\
\text { GII }\end{array}$ & $\begin{array}{c}100 \% \\
(12 / 12)\end{array}$ & (5.00E+06 Max) & $\begin{array}{l}\text { Nordgren } \\
\text { et al., } 2009\end{array}$ \\
\hline Sweden & Noroviruses & $\begin{array}{l}18 \% \\
(4 / 22)\end{array}$ & $\begin{array}{c}3.40 \mathrm{E}+01 \\
(\mathrm{SD} \pm 1.7) \\
(1.50 \mathrm{E}+02 \mathrm{Max})\end{array}$ & $\begin{array}{l}\text { Ottoson et } \\
\text { al., } 2006\end{array}$ \\
\hline US & $\begin{array}{l}\text { Norovirus } \\
\text { GII }\end{array}$ & $\begin{array}{c}0 \% \\
(0 / 8 \square\end{array}$ & NR & $\begin{array}{l}\text { Simmons et } \\
\text { al., } 2011\end{array}$ \\
\hline
\end{tabular}

${ }^{\text {a }}$ GC gene copies; ${ }^{\text {b }}$ SD Standard deviation; NR: Not reported

Norovirus occurrence data are summarized in Table 4. Noroviruses can also be detected in river water samples, and tend to show higher concentration during rain events probably due to sewer overflow (Hata, 2014). Noroviruses GI and GII in river water may reach $10^{3}-10^{4}$ gene copies /L (Haramoto, 2005), but rarely reach $10^{5}$ gene copies /L
(Lodder, 2005; Haramoto, 2012a, 2012b). Sapoviruses are less frequently found in river water samples used as source water for water supply in Japan (Haramoto, 2012a, 2012b), and concentration up to $10^{5}$ gene copies /L have been reported (Sano et al., 2011). Many different sapovirus and norovirus genotypes have been detected in river water samples (Kitajima, 2010a; Kitajima, 2010b) and have a worldwide distribution including developing country such as Kenya (Kiulia, 2010).

Table. 4 Calicivirus concentrations in environmental waters

\begin{tabular}{|c|c|c|c|c|c|}
\hline Area & Sample Type & Virus & $\begin{array}{c}\text { Percent Positive } \\
\text { (\# of Samples) }\end{array}$ & $\begin{array}{c}\text { Concentrations } \\
\text { Average } \\
\text { GC/L } \mathbf{L}^{\mathrm{a}} \\
\text { Mean (Range) } \\
\end{array}$ & Reference \\
\hline Brazil & $\begin{array}{l}\text { Surface } \\
\text { water }\end{array}$ & $\begin{array}{c}\text { Norovirus } \\
\text { GI }\end{array}$ & $\mathrm{NR}^{\mathrm{b}}$ & $\begin{array}{c}(1.71 \mathrm{E}+02 \text { to } \\
8.14 \mathrm{E}+03)\end{array}$ & $\begin{array}{c}\text { Victoria et } \\
\text { al., } 2010\end{array}$ \\
\hline Brazil & $\begin{array}{l}\text { Surface } \\
\text { water }\end{array}$ & $\begin{array}{c}\text { Norovirus } \\
\text { GII }\end{array}$ & NR & $\begin{array}{c}(1.14 \mathrm{E}+02 \text { to } \\
3.72 \mathrm{E}+03)\end{array}$ & $\begin{array}{c}\text { Victoria et } \\
\text { al., } 2010\end{array}$ \\
\hline Egypt & $\begin{array}{l}\text { Surface } \\
\text { water }\end{array}$ & $\begin{array}{c}\text { Norovirus } \\
\text { GI }\end{array}$ & $\begin{array}{l}<32 \% \\
(144)\end{array}$ & $\begin{array}{c}5.30 \mathrm{E}+02 \\
(\mathrm{SD} \pm 2.40 \mathrm{E}+02)\end{array}$ & $\begin{array}{l}\text { El-Senousy } \\
\text { et al., } 2013\end{array}$ \\
\hline Egypt & $\begin{array}{l}\text { Surface } \\
\text { water }\end{array}$ & $\begin{array}{c}\text { Norovirus } \\
\text { GII }\end{array}$ & $\begin{array}{l}<32 \% \\
(144)\end{array}$ & $\begin{array}{c}3.30 \mathrm{E}+02 \\
(\mathrm{SD} \pm 4.30 \mathrm{E}+02)\end{array}$ & $\begin{array}{l}\text { El-Senousy } \\
\text { et al., } 2013\end{array}$ \\
\hline Germany & $\begin{array}{l}\text { Surface } \\
\text { water }\end{array}$ & $\begin{array}{l}\text { Norovirus } \\
\text { GII }\end{array}$ & $\begin{array}{c}32 \% \\
(13 / 41)\end{array}$ & $\begin{array}{c}(9.40 \mathrm{E}+00 \text { to } \\
2.70 \mathrm{E}+04)\end{array}$ & $\begin{array}{c}\text { Hamza et } \\
\text { al., } 2009\end{array}$ \\
\hline Hong Kong & Seawater & $\begin{array}{c}\text { Norovirus } \\
\text { GI }\end{array}$ & $\begin{array}{l}67 \% \\
(4 / 6)\end{array}$ & $\begin{array}{l}(3.90 \mathrm{E}+02 \text { to } \\
2.28 \mathrm{E}+03)\end{array}$ & $\begin{array}{c}\text { Yang et al., } \\
2012\end{array}$ \\
\hline Hong Kong & Seawater & $\begin{array}{c}\text { Norovirus } \\
\text { GII }\end{array}$ & $\begin{array}{c}100 \% \\
(6 / 6)\end{array}$ & $\begin{array}{c}(5.30 \mathrm{E}+02 \text { to } \\
3.68 \mathrm{E}+03)\end{array}$ & $\begin{array}{c}\text { Yang et al., } \\
2012\end{array}$ \\
\hline Italy & Seawater & $\begin{array}{l}\text { Norovirus } \\
\text { GII }\end{array}$ & $\begin{array}{l}12 \% \\
(3 / 26)\end{array}$ & $\begin{array}{c}(7.60 \mathrm{E}+00 \text { to } \\
2.40 \mathrm{E}+02)\end{array}$ & $\begin{array}{l}\text { La Rosa et } \\
\text { al., 2007; } \\
\text { La Rosa et } \\
\text { al., } 2009\end{array}$ \\
\hline Japan & $\begin{array}{l}\text { Surface } \\
\text { water }\end{array}$ & $\begin{array}{c}\text { Norovirus } \\
\text { GI }\end{array}$ & $\begin{array}{c}53 \% \\
(34 / 64)\end{array}$ & $\begin{array}{c}8.70 \mathrm{E}+01 \\
(1.60 \mathrm{E}+03 \mathrm{Max})\end{array}$ & $\begin{array}{l}\text { Haramoto } \\
\text { et al., } 2005\end{array}$ \\
\hline Japan & $\begin{array}{l}\text { Surface } \\
\text { water }\end{array}$ & $\begin{array}{c}\text { Norovirus } \\
\text { GII }\end{array}$ & $\begin{array}{c}44 \% \\
(29 / 64)\end{array}$ & $\begin{array}{c}6.10 \mathrm{E}+02 \\
(7.00 \mathrm{E}+03 \mathrm{Max})\end{array}$ & $\begin{array}{l}\text { Haramoto } \\
\text { et al., } 2005\end{array}$ \\
\hline Japan & $\begin{array}{l}\text { Surface } \\
\text { water }\end{array}$ & Sapovirus & $\begin{array}{c}64 \% \\
(22 / 36)\end{array}$ & $(1.00 \mathrm{E}+02 \mathrm{Max})$ & $\begin{array}{c}\text { Haramoto } \\
\text { et al., } \\
2008 \mathrm{~b}\end{array}$ \\
\hline Japan & $\begin{array}{l}\text { Surface } \\
\text { water }\end{array}$ & $\begin{array}{c}\text { Norovirus } \\
\text { GI }\end{array}$ & $\begin{array}{c}13 \% \\
(8 / 64)\end{array}$ & $\begin{array}{c}(2.70 \mathrm{E}+02 \text { to } \\
3.30 \mathrm{E}+04)\end{array}$ & $\begin{array}{l}\text { Haramoto } \\
\text { et al., } 2012\end{array}$ \\
\hline
\end{tabular}




\begin{tabular}{|c|c|c|c|c|c|}
\hline Area & Sample Type & Virus & $\begin{array}{l}\text { Percent Positive } \\
\text { (\# of Samples) }\end{array}$ & $\begin{array}{c}\text { Concentrations } \\
\text { Average } \\
\text { GC/L } \mathbf{L}^{\mathrm{a}} \\
\text { Mean (Range) }\end{array}$ & Reference \\
\hline Japan & $\begin{array}{l}\text { Surface } \\
\text { water }\end{array}$ & $\begin{array}{l}\text { Norovirus } \\
\text { GII }\end{array}$ & $\begin{array}{c}1 \% \\
(1 / 64)\end{array}$ & $\begin{array}{c}3.40 \mathrm{E}+02 \\
(3.40 \mathrm{E}+02 \text { Max) }\end{array}$ & $\begin{array}{l}\text { Haramoto } \\
\text { et al., } 2012\end{array}$ \\
\hline Japan & $\begin{array}{l}\text { Surface } \\
\text { water }\end{array}$ & Sapovirus & $\begin{array}{c}3 \% \\
(2 / 64)\end{array}$ & $\begin{array}{c}(4.60 \mathrm{E}+02 \text { to } \\
8.20 \mathrm{E}+02)\end{array}$ & $\begin{array}{l}\text { Haramoto } \\
\text { et al., } 2012\end{array}$ \\
\hline Japan & $\begin{array}{l}\text { Surface } \\
\text { water }\end{array}$ & $\begin{array}{l}\text { Norovirus } \\
\text { GIV }\end{array}$ & $\begin{array}{c}31 \% \\
(15 / 48)\end{array}$ & $\begin{array}{c}(1.60 \mathrm{E}+02 \text { to } \\
1.50 \mathrm{E}+04)\end{array}$ & $\begin{array}{l}\text { Kitajima et } \\
\text { al., } 2009\end{array}$ \\
\hline Korea & Groundwater & $\begin{array}{l}\text { Norovirus } \\
\text { GI }\end{array}$ & $\begin{array}{c}0.18 \% \\
(2 / 1090)\end{array}$ & NR & $\begin{array}{l}\text { Lee et al., } \\
2013\end{array}$ \\
\hline Korea & Groundwater & $\begin{array}{l}\text { Norovirus } \\
\text { GII }\end{array}$ & $\begin{array}{c}0.55 \% \\
(6 / 1090)\end{array}$ & NR & $\begin{array}{l}\text { Lee et al., } \\
2013\end{array}$ \\
\hline Netherlands & $\begin{array}{l}\text { Surface } \\
\text { water }\end{array}$ & Noroviruses & $\begin{array}{c}100 \% \\
(8 / 8)\end{array}$ & $\begin{array}{c}(4.00 \mathrm{E}+00 \text { to } \\
4.90 \mathrm{E}+03)\end{array}$ & $\begin{array}{l}\text { Lodder and } \\
\text { Husman, } \\
2005\end{array}$ \\
\hline $\begin{array}{l}\text { New } \\
\text { Zealand }\end{array}$ & $\begin{array}{l}\text { Surface } \\
\text { water }\end{array}$ & $\begin{array}{c}\text { Norovirus } \\
\text { GII }\end{array}$ & $\begin{array}{l}17 \% \\
(1 / 6)\end{array}$ & $2.00 \mathrm{E}+02$ & $\begin{array}{l}\text { Wolf et al., } \\
2010\end{array}$ \\
\hline Singapore & $\begin{array}{l}\text { Surface } \\
\text { water }\end{array}$ & $\begin{array}{c}\text { Norovirus } \\
\text { GI }\end{array}$ & $\begin{array}{c}20 \% \\
(20 / 93)\end{array}$ & $\begin{array}{c}7.00 \mathrm{E}+00 \\
(\mathrm{SD} \pm 9.9 \mathrm{E}+01) \\
(2.16 \mathrm{E}+03 \mathrm{Max})\end{array}$ & $\begin{array}{l}\text { Liang et al., } \\
2015\end{array}$ \\
\hline Singapore & $\begin{array}{l}\text { Surface } \\
\text { water }\end{array}$ & $\begin{array}{c}\text { Norovirus } \\
\text { GII }\end{array}$ & $\begin{array}{c}48 \% \\
(45 / 93)\end{array}$ & $\begin{array}{c}1.04 \mathrm{E}+02 \\
(\mathrm{SD} \pm 2.5 \mathrm{E}+03) \\
(1.55 \mathrm{E}+04 \mathrm{Max})\end{array}$ & $\begin{array}{l}\text { Liang et al., } \\
\quad 2015\end{array}$ \\
\hline Singapore & $\begin{array}{l}\text { Surface } \\
\text { water }\end{array}$ & $\begin{array}{l}\text { Norovirus } \\
\text { GI }\end{array}$ & $\begin{array}{c}25 \% \\
(16 / 65)\end{array}$ & $9.50 \mathrm{E}+01$ & $\begin{array}{l}\text { Rezaeinejad } \\
\text { et al., } 2014\end{array}$ \\
\hline Singapore & $\begin{array}{l}\text { Surface } \\
\text { water }\end{array}$ & $\begin{array}{c}\text { Norovirus } \\
\text { GII }\end{array}$ & $\begin{array}{c}48 \% \\
(31 / 65)\end{array}$ & $3.70 \mathrm{E}+02$ & $\begin{array}{c}\text { Rezaeinejad } \\
\text { et al., } 2014\end{array}$ \\
\hline Thailand & $\begin{array}{l}\text { Surface } \\
\text { water }\end{array}$ & $\begin{array}{l}\text { Norovirus } \\
\text { GII }\end{array}$ & $\begin{array}{c}13 \% \\
(3 / 24)\end{array}$ & $\begin{array}{c}(2.80 \mathrm{E}-01 \text { to } \\
7.90 \mathrm{E}+02)\end{array}$ & $\begin{array}{l}\text { Hata et al., } \\
2011\end{array}$ \\
\hline US & Seawater & $\begin{array}{l}\text { Norovirus } \\
\text { GI }\end{array}$ & $\begin{array}{c}7 \% \\
(5 / 72)\end{array}$ & $(1.40 \mathrm{E}+09 \mathrm{Max})$ & $\begin{array}{l}\text { Gentry et } \\
\text { al., } 2009\end{array}$ \\
\hline US & Seawater & $\begin{array}{c}\text { Norovirus } \\
\text { GII }\end{array}$ & $\begin{array}{c}1 \% \\
(1 / 72)\end{array}$ & $(1.10 \mathrm{E}+02 \mathrm{Max})$ & $\begin{array}{l}\text { Gentry et } \\
\text { al., } 2009\end{array}$ \\
\hline US & Groundwater & $\begin{array}{c}\text { Norovirus } \\
\text { GI }\end{array}$ & $\begin{array}{c}4 \% \\
(48 / 1204)\end{array}$ & $\begin{array}{c}\text { 6.00E-01 } \\
\text { (1.16E+02 Max) }\end{array}$ & $\begin{array}{l}\text { Borchardt } \\
\text { et al., } 2012\end{array}$ \\
\hline
\end{tabular}

Both GI and GII noroviruses were detected in $32 \%$ of irrigation water samples (10 L) collected in Egypt. A study on fresh produce such as lettuce showed only GI norovirus and no norovirus GII (El-Senousy 2013), which may indicate that norovirus GI is more persistent on the surface of fresh produce. More data on the occurrence of norovirus and sapovirus is needed from developing countries in particular in countries where irrigation using untreated wastewater is a common practice.

\subsubsection{Groundwater and drinking water}

Noroviruses and sapoviruses have been detected in groundwater (Table 4). For example, in $500 \mathrm{~L}$ ground water samples used for food-catering facilities in Korea norovirus was positive in only $0.64 \%$ tested (Lee et al., 2013), which is less than nationwide groundwater survey in Korea showing $8.7 \%$ positive (Lee et al., 2012). Also in a study from the US, $4 \%$ of groundwater samples used for tap water contained norovirus genomes with concentrations as high as 116 gene copies /L of norovirus GI (Borchardt, 2012).
Limited data are available on the presence of noroviruses in drinking water. A study in Japan found that $4 \%$ and $7 \%$ was positive for norovirus GI and GII respectively under the effort using whole concentrates onto RT-PCR detection from 100-532 liters of tap water. However, most of these samples contained more than 0.5 $\mathrm{mg} / \mathrm{L}$ of chlorine and therefore the detected norovirus was most likely not infectious. In a report from Spain, no norovirus was detected in tap water (Pérez-Sautu 2012). Waterborne norovirus outbreaks are reported and they can often be linked to a sewage-contaminated drinking water (Bitler et al., 2013)

\subsubsection{Seawater and shellfish}

Coastal seawaters are affected strongly by treated/untreated urban sewer discharge and also by combined sewer overflow during rain events. The concentration of norovirus in seawater varies greatly ranging from $10^{2}$ to $10^{3}$ gene copies / L in Brazil (Victoria, 2010), whereas a coastal water sample in a study in the US contained $10^{9}$ gene copies /L (Gentry, 2009). Oysters in 
England and Wales from cold waters $\left(<5^{\circ} \mathrm{C}\right)$ had significantly higher norovirus content than those from warmer waters $\left(>10^{\circ} \mathrm{C}\right)$, which may be due to the seasonal prevalence of the virus in the community or linked with oyster metabolic function (Campos et al., 2017).

Approximately 9\% of the shellfish samples sold in the markets in France tested positive for norovirus (Schaeffer, 2013), whereas up to $90 \%$ of the oysters from harvesting areas in the United Kingdom tested positive with a higher positive ratio in the winter than in summer (Lowther, 2012). Those differences suggest that timing of harvesting and depuration of the oyster is important before the oysters are sold commercially. Ueki et al described the detection of the same norovirus genotype in patients, oysters, and from samples collected at the wastewater treatment plants (Ueki, 2005). Since noroviruses bound to oyster digestive tissues (Le Guyader et al., 2006) and norovirus genomes were not bioaccumulated (Dancer, 2010), detected genomes from oysters are likely to be originated from intact virus particles.
In shellfish from Morocco, sapoviruses were less frequently found than noroviruses but at higher levels than other enteric viruses (Benabbes, 2013).

\subsection{Persistence}

Limited data are available on the persistence and survival of noroviruses and sapoviruses except for a few human challenge studies. Several cultivable surrogate viruses including murine norovirus and Tulane virus are often used for persistence studies, whereas feline calicivirus had been used but is less reliable due to its vulnerability to acid and base condition (Cannon et al., 2006). Norovirus GII and MNV showed long persistence (T90 > 21 days at $25^{\circ} \mathrm{C}$ or cooler) in urine, in wastewater or in biofilms (Table 5). Published persistence and survival data show days of survival on the surface based on the surrogate study using murine norovirus, much longer persistency based on the RT-qPCR detection, and extremely long ( $>1,000$ days) persistence under suitable condition such as in groundwater(Tables 6, 7, and 8).

Table. 5 Persistence of Calicivirus in urine, wastewater and biofilms

\begin{tabular}{|c|c|c|c|c|c|}
\hline Area & Virus & $\begin{array}{c}\text { T90 } \\
\text { (Days) }^{\mathrm{a}}\end{array}$ & $\begin{array}{c}\text { Temperature } \\
{ }^{\circ} \mathrm{C}\end{array}$ & $\begin{array}{c}\text { Sample } \\
\text { Type }\end{array}$ & Reference \\
\hline $\begin{array}{l}\text { Burkina } \\
\text { Faso }\end{array}$ & $\mathrm{MNV}^{\mathrm{a}}$ & 42 & 15 & Urine & Makaya et al., 2015 \\
\hline $\begin{array}{l}\text { Burkina } \\
\text { Faso }\end{array}$ & MNV & 21 & 25 & Urine & Makaya et al., 2015 \\
\hline $\begin{array}{l}\text { Burkina } \\
\text { Faso }\end{array}$ & MNV & 3.5 & 42 & Urine & Makaya et al., 2015 \\
\hline Luxembourg & Norovirus GII ${ }^{\mathrm{a}}$ & $>49$ & 4 & Wastewater & Skraber et al., 2009 \\
\hline Luxembourg & Norovirus GII & 21 & 20 & Wastewater & Skraber et al., 2009 \\
\hline Luxembourg & Norovirus GII & $>49$ & 4 & Biofilm & Skraber et al., 2009 \\
\hline Luxembourg & Norovirus GII & $>49$ & 20 & Biofilm & Skraber et al., 2009 \\
\hline
\end{tabular}

Table. 6 Persistence of Caliciviruses in PBS and Ground water

\begin{tabular}{|c|c|c|c|c|c|}
\hline Area & $\begin{array}{c}\text { Sample } \\
\text { Type }\end{array}$ & Virus & T90 (Days) & $\begin{array}{c}\text { Temperature } \\
{ }^{\circ} \mathrm{C} \\
\end{array}$ & Reference \\
\hline $\begin{array}{l}\text { Burkina } \\
\text { Faso }\end{array}$ & PBS & $\mathrm{MNV}^{\mathrm{a}, \mathrm{b}}$ & 28 & 15 & Makya et al., 2015 \\
\hline $\begin{array}{l}\text { Burkina } \\
\text { Faso }\end{array}$ & PBS & $\mathrm{MNV}^{\mathrm{a}, \mathrm{b}}$ & 12 & 25 & Makaya et al., 2015 \\
\hline $\begin{array}{l}\text { Burkina } \\
\text { Faso }\end{array}$ & PBS & $\mathrm{MNV}^{\mathrm{a}, \mathrm{b}}$ & $42^{c}$ & 42 & Makaya et al.. 2015 \\
\hline
\end{tabular}




\begin{tabular}{|c|c|c|c|c|c|}
\hline Area & $\begin{array}{l}\text { Sample } \\
\text { Type }\end{array}$ & Virus & $\begin{array}{c}\text { T90 } \\
\text { (Days) }\end{array}$ & $\underset{{ }^{\circ} \mathrm{C}}{\text { Temperature }}$ & Reference \\
\hline USA & Groundwater & $\begin{array}{l}\text { Norovirus GI } \\
\text { Norwalk }^{\mathrm{c}}\end{array}$ & 1151 & $\mathrm{RT}^{\mathrm{d}}$ & $\begin{array}{c}\text { Seitz et } \\
\text { al., } 2011\end{array}$ \\
\hline USA & Groundwater & $\begin{array}{l}\text { Norovirus GI } \\
\text { Norwalk }^{c}\end{array}$ & 707 & RT & $\begin{array}{l}\text { Seitz et } \\
\text { al., } 2011\end{array}$ \\
\hline USA & PBS & $\begin{array}{l}\text { Norovirus GI } \\
\text { Norwalk }^{\mathrm{e}}\end{array}$ & 120 & 4 & $\begin{array}{l}\text { Liu et al., } \\
2012\end{array}$ \\
\hline USA & PBS & $\begin{array}{l}\text { Norovirus GI } \\
\text { Norwalk }^{\mathrm{e}}\end{array}$ & 35 & RT & $\begin{array}{l}\text { Liu et al., } \\
2012\end{array}$ \\
\hline USA & PBS & $\begin{array}{l}\text { Norovirus GI } \\
\text { Norwalk }^{\mathrm{e}}\end{array}$ & 5.5 & 37 & $\begin{array}{l}\text { Liu et al., } \\
2012\end{array}$ \\
\hline
\end{tabular}

Table. 7 Persistence of Calicivirus in foods

\begin{tabular}{|c|c|c|c|c|c|c|}
\hline Area & Virus & $\begin{array}{c}\text { T90 } \\
\text { (Days) }\end{array}$ & Temperature & Method & Matrix & Reference \\
\hline Korea & $\mathrm{MNV}^{\mathrm{a}}$ & 8.18 & 10 & $\begin{array}{l}\text { Cell } \\
\text { culture }\end{array}$ & $\begin{array}{l}\text { Soy source } \\
5 \% \mathrm{NaCl}\end{array}$ & $\begin{array}{l}\text { Park and } \\
\text { Ha, } 2015\end{array}$ \\
\hline Korea & MNV & 4.68 & 10 & $\begin{array}{c}\text { Cell } \\
\text { culture }\end{array}$ & $\begin{array}{l}\text { Soy source } \\
10 \% \mathrm{NaCl}\end{array}$ & $\begin{array}{l}\text { Park and } \\
\text { Ha, } 2015\end{array}$ \\
\hline Korea & MNV & 3.39 & 10 & $\begin{array}{c}\text { Cell } \\
\text { culture }\end{array}$ & $\begin{array}{c}\text { Soy source } \\
15 \% \mathrm{NaCl}\end{array}$ & $\begin{array}{l}\text { Park and } \\
\text { Ha, } 2015\end{array}$ \\
\hline Korea & MNV & 2.96 & 10 & $\begin{array}{c}\text { Cell } \\
\text { culture }\end{array}$ & $\begin{array}{l}\text { Soy source } \\
20 \% \mathrm{NaCl}\end{array}$ & $\begin{array}{l}\text { Park and } \\
\text { Ha, } 2015\end{array}$ \\
\hline Netherlands & MNV & 7 & 10 & $\begin{array}{c}\text { Cell } \\
\text { FXOXXuH }\end{array}$ & Strawberry & $\begin{array}{l}\text { Verhaelen } \\
\text { et al., } 2012\end{array}$ \\
\hline Netherlands & MNV & 3 & 21 & $\begin{array}{l}\text { Cell } \\
\text { FXOXuH }\end{array}$ & Raspberry & $\begin{array}{c}\text { Verhaelen } \\
\text { et al., } 2012\end{array}$ \\
\hline Netherlands & MNV & 3 & 21 & Real-time PCR & Strawberry & $\begin{array}{l}\text { Verhaelen } \\
\text { et al., } 2012\end{array}$ \\
\hline Netherlands & MNV & 1 & 21 & $\begin{array}{l}\text { Cell } \\
\text { FXOXuH }\end{array}$ & Strawberry & $\begin{array}{r}\text { Verhaelen } \\
\text { et al.,2012 }\end{array}$ \\
\hline Netherlands & Noroviru GI.4 & 2 & 21 & Real-time PCR & Strawberry & $\begin{array}{l}\text { Verhaelen } \\
\text { et al.,2012 }\end{array}$ \\
\hline USA & $\begin{array}{l}\text { Norovirus GI } \\
\text { Norwalk }\end{array}$ & 16.6 & 4 & $\begin{array}{l}\text { Real-time } \\
\text { PCR }\end{array}$ & Lettuce & $\begin{array}{r}\text { Escudero } \\
\text { et al., } 2012\end{array}$ \\
\hline USA & $\begin{array}{c}\text { Norovirus GI } \\
\text { Norwalk }\end{array}$ & 9 & 22 & $\begin{array}{l}\text { Real-time } \\
\text { PCR }\end{array}$ & Lettuce & $\begin{array}{l}\text { Escudero } \\
\text { et al., } 2012\end{array}$ \\
\hline
\end{tabular}




\begin{tabular}{|c|c|c|c|c|c|c|}
\hline Area & T90 & (Days) & $\begin{array}{c}\text { Temperature } \\
{ }^{\circ} \mathrm{C} \\
\end{array}$ & Method & Matrix & Reference \\
\hline USA & $\begin{array}{c}\text { Norovirus GII } \\
\text { Snow Mountain }\end{array}$ & 25 & 4 & $\begin{array}{l}\text { Real-time } \\
\text { PCR }\end{array}$ & Lettuce & $\begin{array}{c}\text { Escudero } \\
\text { et al., } 2012\end{array}$ \\
\hline USA & $\begin{array}{l}\text { Norovirus GII } \\
\text { Snow Mountain }\end{array}$ & 16.6 & 22 & $\begin{array}{l}\text { Real-time } \\
\text { PCR }\end{array}$ & Lettuce & $\begin{array}{l}\text { Escudero } \\
\text { HMDQ⿴囗⿰丨丨⿱⺊口灬 } 012\end{array}$ \\
\hline USA & MNV & 107 & $\mathrm{RTb}$ & $\begin{array}{l}\text { Real-time } \\
\text { PCR }\end{array}$ & Lettuce & $\begin{array}{l}\text { Escudero } \\
\text { et al., } \\
2012\end{array}$ \\
\hline USA & MNV & 333 & 4 & $\begin{array}{l}\text { Real-time } \\
\text { PCR }\end{array}$ & Lettuce & $\begin{array}{l}\text { Escudero } \\
\text { et al., } \\
2012\end{array}$ \\
\hline USA & MNV & 4.5 & RT & $\begin{array}{l}\text { Plaque } \\
\text { assay }\end{array}$ & $\begin{array}{c}\text { Lettuce + } \\
\text { Artificial } \\
\text { feces }\end{array}$ & $\begin{array}{l}\text { Escudero } \\
\text { et al., } \\
2012\end{array}$ \\
\hline USA & MNV & 11 & 4 & $\begin{array}{l}\text { Plaque } \\
\text { assay }\end{array}$ & $\begin{array}{c}\text { Lettuce + } \\
\text { Artificial } \\
\text { feces }\end{array}$ & $\begin{array}{c}\text { Escudero } \\
\text { et al., } \\
2012\end{array}$ \\
\hline USA & MNV & 4.84 & 18 & $\begin{array}{l}\text { Plaque } \\
\text { assay }\end{array}$ & $\begin{array}{l}\text { Spinach } \\
\text { semi-savoy } \\
\text { whole }\end{array}$ & $\begin{array}{l}\text { Hirneisen } \\
\text { and Kniel, } \\
2013\end{array}$ \\
\hline USA & MNV & 2.55 & 18 & $\begin{array}{l}\text { Plaque } \\
\text { assay }\end{array}$ & $\begin{array}{l}\text { Spinach } \\
\text { semi-savoy, } \\
\text { adaxial }\end{array}$ & $\begin{array}{l}\text { Hirneisen } \\
\text { and Kniel, } \\
2013\end{array}$ \\
\hline USA & MNV & 3.77 & 18 & $\begin{array}{l}\text { Plaque } \\
\text { assay }\end{array}$ & $\begin{array}{l}\text { Spinach } \\
\text { semi-savoy, } \\
\text { adaxial }\end{array}$ & $\begin{array}{l}\text { Hirneisen } \\
\text { and Kniel, } \\
2013\end{array}$ \\
\hline USA & MNV & 2.5 & 18 & $\begin{array}{l}\text { Plaque } \\
\text { assay }\end{array}$ & $\begin{array}{l}\text { Spinach } \\
\text { smooth, } \\
\text { whole }\end{array}$ & $\begin{array}{l}\text { Hirneisen } \\
\text { and Kniel, } \\
2013\end{array}$ \\
\hline USA & MNV & 1.94 & 18 & $\begin{array}{l}\text { Plaque } \\
\text { assay }\end{array}$ & $\begin{array}{l}\text { Spinach } \\
\text { smooth, } \\
\text { adaxial }\end{array}$ & $\begin{array}{c}\text { Hirneisen } \\
\text { and Kniel, } \\
2013\end{array}$ \\
\hline USA & MNV & 2.71 & 18 & $\begin{array}{l}\text { Plaque } \\
\text { assay }\end{array}$ & $\begin{array}{l}\text { Spinach } \\
\text { smooth, } \\
\text { adaxial }\end{array}$ & $\begin{array}{l}\text { Hirneisen } \\
\text { and Kniel, } \\
2013\end{array}$ \\
\hline USA & $\begin{array}{l}\text { Tulane } \\
\text { Virus }\end{array}$ & 5.73 & 18 & $\begin{array}{l}\text { Plaque } \\
\text { assay }\end{array}$ & $\begin{array}{l}\text { Spinach } \\
\text { semi-savoy } \\
\text { whole }\end{array}$ & $\begin{array}{l}\text { Hirneisen } \\
\text { and Kniel, } \\
2013\end{array}$ \\
\hline USA & $\begin{array}{l}\text { Tulane } \\
\text { Virus }\end{array}$ & 2.61 & 18 & $\begin{array}{l}\text { Plaque } \\
\text { assay }\end{array}$ & $\begin{array}{l}\text { Spinach } \\
\text { semi-savoy, } \\
\text { adaxial }\end{array}$ & $\begin{array}{l}\text { Hirneisen } \\
\text { and Kniel, } \\
2013\end{array}$ \\
\hline USA & $\begin{array}{l}\text { Tulane } \\
\text { Virus }\end{array}$ & 4.38 & 18 & $\begin{array}{l}\text { Plaque } \\
\text { assay }\end{array}$ & $\begin{array}{l}\text { Spinach } \\
\text { semi-savoy, } \\
\text { adaxial }\end{array}$ & $\begin{array}{l}\text { Hirneisen } \\
\text { and Kniel, } \\
2013\end{array}$ \\
\hline USA & $\begin{array}{l}\text { Tulane } \\
\text { Virus }\end{array}$ & 3.07 & 18 & $\begin{array}{l}\text { Plaque } \\
\text { assay }\end{array}$ & $\begin{array}{l}\text { Spinach } \\
\text { smooth, } \\
\text { whole }\end{array}$ & $\begin{array}{l}\text { Hirneisen } \\
\text { and Kniel, } \\
2013\end{array}$ \\
\hline USA & $\begin{array}{l}\text { Tulane } \\
\text { Virus }\end{array}$ & 2.25 & 18 & $\begin{array}{l}\text { Plaque } \\
\text { assay }\end{array}$ & $\begin{array}{l}\text { Spinach } \\
\text { smooth, } \\
\text { adaxial }\end{array}$ & $\begin{array}{l}\text { Hirneisen } \\
\text { and Kniel, } \\
2013\end{array}$ \\
\hline USA & $\begin{array}{l}\text { Tulane } \\
\text { Virus }\end{array}$ & 2.24 & 18 & $\begin{array}{l}\text { Plaque } \\
\text { assay }\end{array}$ & $\begin{array}{l}\text { Spinach } \\
\text { smooth, } \\
\text { adaxial }\end{array}$ & $\begin{array}{c}\text { Hirneisen } \\
\text { and Kniel, } \\
2013\end{array}$ \\
\hline
\end{tabular}


Table. 8 Persistence of Calicivirus on fomites

\begin{tabular}{|c|c|c|c|c|c|}
\hline Area & Virus & $\begin{array}{c}\text { T90 } \\
\text { (Days) }\end{array}$ & $\begin{array}{c}\text { Temperature } \\
{ }^{\circ} \mathrm{C}\end{array}$ & Sample Type & Reference \\
\hline France & $\mathrm{MNV}^{\mathrm{a}, \mathrm{b}}$ & 4.3 & 9 & Glass, humidity 10־ & Noue et al., 2014 \\
\hline France & $\mathrm{MNV}^{\mathrm{a}, \mathrm{b}}$ & 3.3 & 9 & Glass, humidity 35— & Noue et al., 2014 \\
\hline France & $\mathrm{MNV}^{\mathrm{a}, \mathrm{b}}$ & 1.4 & 9 & Glass, humidity 55 & Noue et al., 2014 \\
\hline France & $\mathrm{MNV}^{\mathrm{a}, \mathrm{b}}$ & 3.4 & 9 & 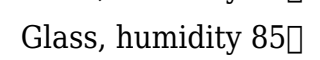 & Noue et al., 2014 \\
\hline France & $\mathrm{MNV}^{\mathrm{a}, \mathrm{b}}$ & 3.7 & 9 & Glass, humidity $100 \square$ & Noue et al., 2014 \\
\hline France & $\mathrm{MNV}^{\mathrm{a}, \mathrm{b}}$ & 2.8 & 25 & Glass, humidity 10๑ & Noue et al., 2014 \\
\hline France & $\mathrm{MNV}^{\mathrm{a}, \mathrm{b}}$ & 1 & 25 & Glass, humidity 35๑ & Noue et al., 2014 \\
\hline France & $\mathrm{MNV}^{\mathrm{a}, \mathrm{b}}$ & $<0.42$ & 25 & Glass, humidity 55ロ & Noue et al., 2014 \\
\hline France & $\mathrm{MNV}^{\mathrm{a}, \mathrm{b}}$ & $<0.42$ & 25 & Glass, humidity 85— & Noue et al., 2014 \\
\hline France & $\mathrm{MNV}^{\mathrm{a}, \mathrm{b}}$ & 0.58 & 25 & Glass, humidity $100 \square$ & Noue et al., 2014 \\
\hline Korea & $\mathrm{MNV}^{\mathrm{b}}$ & 4.8 & 15 & Wood, $30 \%$ humidity & Kim et al., 2012 \\
\hline Korea & $\mathrm{MNV}^{\mathrm{b}}$ & 7.2 & 15 & Wood, $50 \%$ humidity & Kim et al., 2012 \\
\hline Korea & $\mathrm{MNV}^{\mathrm{b}}$ & 6.6 & 15 & Wood, $70 \%$ humidity & Kim et al., 2012 \\
\hline Korea & $\mathrm{MNV}^{\mathrm{b}}$ & 10.2 & 25 & Wood, $30 \%$ humidity & Kim et al., 2012 \\
\hline Korea & $\mathrm{MNV}^{\mathrm{b}}$ & 7.2 & 25 & Wood, $50 \%$ humidity & Kim et al., 2012 \\
\hline Korea & $\mathrm{MNV}^{\mathrm{b}}$ & 2.4 & 25 & Wood, $70 \%$ humidity & Kim et al., 2012 \\
\hline Korea & $\mathrm{MNV}^{\mathrm{b}}$ & 3.2 & 32 & Wood, $30 \%$ humidity & Kim et al., 2012 \\
\hline Korea & $\mathrm{MNV}^{\mathrm{b}}$ & 0.48 & 32 & Wood, $50 \%$ humidity & Kim et al., 2012 \\
\hline Korea & $\mathrm{MNV}^{\mathrm{b}}$ & 1.6 & 32 & Wood, $70 \%$ humidity & Kim et al., 2012 \\
\hline Korea & $\mathrm{MNV}^{\mathrm{b}}$ & 1.12 & 40 & Wood, 30\% humidity & Kim et al., 2012 \\
\hline Korea & $\mathrm{MNV}^{\mathrm{b}}$ & 0.48 & 40 & Wood, $50 \%$ humidity & Kim et al., 2012 \\
\hline Korea & $\mathrm{MNV}^{\mathrm{b}}$ & 0.08 & 40 & Wood, $70 \%$ humidity & Kim et al., 2012 \\
\hline Korea & $\mathrm{MNV}^{\mathrm{b}}$ & 0.54 & 15 & Steel, 30\% humidity & Kim et al., 2012 \\
\hline Korea & $\mathrm{MNV}^{\mathrm{b}}$ & 0.6 & 15 & Steel, 50\% humidity & Kim et al., 2012 \\
\hline Korea & $\mathrm{MNV}^{\mathrm{b}}$ & 0.6 & 15 & Steel, 70\% humidity & Kim et al., 2012 \\
\hline Korea & $\mathrm{MNV}^{\mathrm{b}}$ & 3.2 & 25 & Steel, $30 \%$ humidity & Kim et al., 2012 \\
\hline Korea & $\mathrm{MNV}^{\mathrm{b}}$ & 1.26 & 25 & Steel, 50\% humidity & Kim et al., 2012 \\
\hline Korea & $\mathrm{MNV}^{\mathrm{b}}$ & 0.22 & 25 & Steel, 70\% humidity & Kim et al., 2012 \\
\hline Korea & $\mathrm{MNV}^{\mathrm{b}}$ & 0.08 & 32 & Steel, $30 \%$ humidity & Kim et al., 2012 \\
\hline Korea & $\mathrm{MNV}^{\mathrm{b}}$ & 0.06 & 32 & Steel, 50\% humidity & Kim et al., 2012 \\
\hline Korea & $\mathrm{MNV}^{\mathrm{b}}$ & 0.12 & 32 & Steel, $70 \%$ humidity & Kim et al., 2012 \\
\hline Korea & $\mathrm{MNV}^{\mathrm{b}}$ & 0.07 & 40 & Steel, 30\% humidity & Kim et al., 2012 \\
\hline Korea & $\mathrm{MNV}^{\mathrm{b}}$ & 0.07 & 40 & Steel, 50\% humidity & Kim et al., 2012 \\
\hline Korea & $\mathrm{MNV}^{\mathrm{b}}$ & 0.08 & 40 & Steel, $70 \%$ humidity & Kim et al., 2012 \\
\hline Korea & $\mathrm{MNV}^{\mathrm{b}, \mathrm{c}}$ & 11 & $\mathrm{RT}^{\mathrm{e}}$ & Ceramic & Kim et al., 2014 \\
\hline Korea & $\mathrm{MNV}^{\mathrm{b}, \mathrm{c}}$ & 20.5 & $\mathrm{RT}$ & Wood & Kim et al., 2014 \\
\hline Korea & $\mathrm{MNV}^{\mathrm{b}, \mathrm{c}}$ & 7.23 & $\mathrm{RT}$ & Rubber & Kim et al., 2014 \\
\hline Korea & $\mathrm{MNV}^{\mathrm{b}, \mathrm{c}}$ & 4.05 & RT & Glass & Kim et al., 2014 \\
\hline Korea & $\mathrm{MNV}^{\mathrm{b}, \mathrm{c}}$ & 3.82 & $\mathrm{RT}$ & Stainless steel & Kim et al., 2014 \\
\hline Korea & $\mathrm{MNV}^{\mathrm{b}, \mathrm{c}}$ & 5.74 & $\mathrm{RT}$ & Plastic & Kim et al., 2014 \\
\hline $\mathrm{USA}^{\mathrm{b}}$ & $\begin{array}{l}\text { Norovirus GI } \\
\text { Norwalk }\end{array}$ & 9.1 & 4 & Stainless steel & Liu et al, 2012 \\
\hline $\mathrm{USA}^{\mathrm{b}}$ & $\begin{array}{l}\text { Norovirus GI } \\
\text { Norwalk }\end{array}$ & 3.4 & 37 & Stainless steel & Liu et al, 2012 \\
\hline $\mathrm{USA}^{\mathrm{b}}$ & $\begin{array}{l}\text { Norovirus GI } \\
\text { Norwalk }\end{array}$ & 4.8 & $\mathrm{RT}$ & Stainless steel & Liu et al, 2012 \\
\hline USA & $\begin{array}{l}\text { Norovirus GI } \\
\text { Norwalk }^{\mathrm{d}}\end{array}$ & 28.5 & $\mathrm{RT}$ & Formica & Escudero et al., 2012 \\
\hline
\end{tabular}




\begin{tabular}{|c|c|c|c|c|c|}
\hline Area & Virus & $\begin{array}{c}\text { T90 } \\
\text { (Days) }\end{array}$ & $\begin{array}{c}\text { Temperature } \\
{ }^{\circ} \mathrm{C} \\
\end{array}$ & Sample Type & Reference \\
\hline USA & $\begin{array}{l}\text { Norovirus GI } \\
\text { Norwalk }^{\mathrm{d}}\end{array}$ & 31.3 & $\mathrm{RT}$ & Stainless steel & Escudero et al., 2012 \\
\hline USA & $\begin{array}{l}\text { Norovirus GI } \\
\text { Norwalk }^{\mathrm{d}}\end{array}$ & 34.4 & RT & Ceramic Tile & Escudero et al., 2012 \\
\hline USA & $\begin{array}{c}\text { Norovirus GII } \\
\text { Snow Mountain }^{\mathrm{d}}\end{array}$ & 33.3 & RT & Formica & Escudero et al., 2012 \\
\hline USA & $\begin{array}{c}\text { Norovirus GII } \\
\text { Snow Mountain }^{d}\end{array}$ & 33.3 & RT & ceramic tile & Escudero et al., 2012 \\
\hline USA & $\begin{array}{l}\text { Norovirus GII } \\
\text { Snow Mountain }^{\mathrm{d}}\end{array}$ & 43.4 & RT & Stainless steel & Escudero et al., 2012 \\
\hline USA & $\mathrm{MNV}^{\mathrm{a}}$ & 2.3 & RT & Formica & Escudero et al., 2012 \\
\hline USA & $\mathrm{MNV}^{\mathrm{a}}$ & 4 & RT & Ceramic tile & Escudero et al., 2012 \\
\hline USA & $\mathrm{MNV}^{\mathrm{a}}$ & 4.3 & RT & Stainless steel & Escudero et al., 2012 \\
\hline USA & $\mathrm{MNV}^{\mathrm{a}}$ & 5.2 & RT & Ceramic tile + Artificial feces & Escudero et al., 2012 \\
\hline USA & $\mathrm{MNV}^{\mathrm{a}}$ & 5.8 & RT & Stainless steel+artificial feces & Escudero et al., 2012 \\
\hline USA & $\mathrm{MNV}^{\mathrm{a}}$ & 5.8 & RT & Formica + Artificial feces & Escudero et al., 2012 \\
\hline USA & $\mathrm{MNV}^{\mathrm{d}}$ & 50 & RT & Ceramic tile & Escudero et al., 2012 \\
\hline USA & $\mathrm{MNV}^{\mathrm{d}}$ & 62.5 & RT & Stainless steel & Escudero et al., 2012 \\
\hline USA & $\mathrm{MNV}^{\mathrm{d}}$ & 83 & RT & Formica & Escudero et al., 2012 \\
\hline
\end{tabular}

${ }^{a}$ Murine Norovirus; ${ }^{b}$ Plaque assay; ${ }^{c}$ use of the Weibull model; ${ }^{d}$ Real-time PCR; ${ }^{e}$ RT: Room Temperature

Survival of noroviruses have been studied using human volunteers as a read-out. In one study groundwater was seeded with Norwalk virus (GI.1) and fed to human volunteers which showed that up to 61 days of storage of the seeded groundwater at room temperature in the dark, was still able to infect volunteers (Seitz, 2011). In an older outbreak study, two carpet fitters who removed a carpet in a ward where an outbreak had occurred 3 weeks earlier, got norovirus gastroenteritis symptoms (Cheesbrough, 1997), indicating that virus on carpets may be viable virus for at least 12 days. Similar findings have also been reported in an outbreak in a hotel (Kimura et al., 2011). No data on the persistence of sapovirus have been published.

\subsection{Reductions by Sanitation Management}

\subsection{Wastewater treatment}

In waste stabilization ponds, noroviruses were not effectively removed compared to other wastewater treatment systems (Da Silva et al., 2007). Although it is not quantitative enough due to limited number of data or left censored data sets, norovirus GI and GII were removed up to $1.8 \log _{10}$ and $1.6 \log _{10}$ by waste stabilization pond with a residence time of several weeks (Da Silva et al., 2008), and norovirus GII was removed about $1 \log _{10}$ in a study in Ghana (Silverman et al., 2013).

No data are available on observable norovirus or caliciviruses removals by pit Latrines, vault toilets or dry toilets, septic tanks, wetlands, aerated lagoons. Thus other RNA viruses and coliphages that serve as surrogates for the human caliciviruses may best represent the efficiency of removal.

\subsubsection{Composting of fecal wastes}

MNV RNA could not be detected after 1 day at $65^{\circ} \mathrm{C}$ in dairy manure compost, while at $60^{\circ} \mathrm{C}$ for 3 days, $3 \log _{10}$ reduction was observed (Wei, 2010). However, infectivity data were not reported.

\subsubsection{Wastewater Treatment Facilities}

In sewer treatment systems, the primary treatment of simple sedimentation did not show removal of viruses (Simmons, 2011). However, noroviruses are removed in secondary treatments, as systematically reviewed (Sano, 2016). Norovirus $\log _{10}$ removal by activated sludge systems is estimated to be 1.48 (95\% confidence interval (CI): $0.96-2.00$ ) and 1.35 (95\% CI: 0.52-2.18) for norovirus GI and GII, respectively. Sapovirus are often negative in treated wastewater as shown in Table 3 so that removal efficiency tend to be difficult to determine, but norovirus and sapovirus showed similar removal by trickling filters to the activated sludge systems (Kitajima, 2014).

It is noteworthy that norovirus genomes and bacteriophages are more persistent in biofilms of wastewater treatment (moving-bed biofilm reactor) than in wastewater (Skraber, 2009), indicating a possible harbor for norovirus during non-endemic period.

Membrane bioreactors (MBR) have become more 
popular in recent years due to its stable treatment of wastewater including its ability to remove viruses. MBR showed better removal of noroviruses than conventional activated sludge systems (Da Silva, 2007), where removal by membrane (Sina et al., 2011) and adsorption to suspended solids (Miura, 2015) play important roles. Sapovirus was effectively $\left(>2 \log _{10}\right.$ ) removed by MBR (Miura et al., 2015). $\log _{10}$ removal efficiency of norovirus GII by MBR is estimated from meta-analysis to be 3.35 (95\% CI: 2.39-4.30) (Sano, 2016).

\subsubsection{Other water treatments}

Coagulation treatment using aluminum sulfate as coagulant, reduced norovirus by $1.5 \log _{10}$ which is similar to MS2 or poliovirus (Shin and Sobsey, 2015), where a river water used for source of tap water is used as test water; $\mathrm{pH}$ 6.6-7.0, turbidity 1.5-2.6 NTU, alkalinity $21-24 \mathrm{mg} / \mathrm{L}$ as $\mathrm{CaCO} 3$, and hardness $20 \mathrm{mg} / \mathrm{L}$ as $\mathrm{CaCO} 3$.

Using river water as the source water, ultrafilters (UFs) were able to remove 1 to $4 \log _{10}$ of norovirus virus-like particles depending on pore size, while almost no reduction was observed by simple application of microfilters (MF) at pore sizes of $0.1 \mathrm{um}$ regardless of the material of filter (PVDF, polyvinylidene difluoride; PTFE, polytetrafluoroethylene; or MC, mixture of cellulose acetate and cellulose nitrate). However, addition of coagulant increased the removal efficiency of MF dramatically, reaching up to more than $4 \log _{10}$, exceeding the removal of UFs (Matsushita, 2013).

\subsection{Disinfection}

\subsubsection{Chlorine and ozone}

One of the first studies looking at the effect of chlorine on Norwalk virus resulted in some surprising data on the resistance of this virus suggesting that noroviruses may be more resistance to chlorine compared to other enteric viruses (Keswick, 1985). However, the experimental design of this study has been questioned as the virus purification methods and dispersion methods were not well documented, nor the quantity of virus was not described in detail. More recent work using RT-PCR for the detection of norovirus RNA, clearly demonstrated that norovirus genomes are reduced $3 \log _{10}$ after $10 \mathrm{~min}$ at $1 \mathrm{mg} / \mathrm{L}$ of chlorine, at $\mathrm{pH} 6,5$, which shows similar resistance to chlorine to other enteric viruses (Shin and Sobsey, 2008).

Treatment by ozone showed that Norwalk virus as well as other enteric viruses can be reduced rapidly and extensively by ozone disinfection (Shin and Sobsey, 2003). No data are available on sapovirus.

\subsubsection{Solar irradiation and UV disinfection}

Solar irradiation may be one of the best options to reduce viruses under limited resources, yet no data are available on noroviruses inactivation due to the lack of an available cell culture method. MNV showed no reduction by irradiation of UV-A, which is the main UV right reaching on the surface of earth, indicating that solar radiation may not be sufficiently effective against noroviruses (Harding and Schwab, 2012).

A few studies have investigated the effect of ultraviolet irradiation on noroviruses but, since no cultivation method is available, only MNV data are available so far, which showed no evidence of resistance higher than other (cultivable) enteric viruses (Lee and Ko, 2013). It is noteworthy that PCR data may overestimate the persistence of noroviruses after inactivation by UV (Rönnqvist, 2014); norovirus genome reduced only 0.62 $\log _{10}$ at $300 \mathrm{~mJ} / \mathrm{cm} 2$ by low-pressure mercury-vapor UV lamp, whereas MNV infectivity reduced $5 \log _{10}$ at $90 \mathrm{~mJ} / \mathrm{cm} 2$. 


\section{References}

Ahmed, S.M., Hall, A.J., Robinson, A.E., Verhoef, L., Premkumar, P., Parashar, U.D. et al. (2014). Global prevalence of norovirus in cases of gastroenteritis: a systematic review and meta-analysis. The Lancet Infectious Diseases. 14, pp. 725-730. doi: 10.1016/S1473-3099(14)70767-4.

Ajami, N., Koo, H., Darkoh, C., Atmar, R.L., Okhuysen, P.C., Jiang, Z.D. et al. (2010). Characterization of NorovirusAssociated Traveler's Diarrhea. Clinical Infectious Diseases. 51, pp. 123-130. doi: 10.1086/653530.

Atmar, R.L., Opekun, A.R., Gilger, M.A., Estes, M.K., Crawford, S.E., Neill, F.H. et al. (2008). Norwalk virus shedding after experimental human infection. Emerging Infectious Diseases. 14, pp. 1553-1557. doi: 10.3201/eid1410.080117.

Barclay, L., Park, G.W., Vega, E., Hall, A., Parashar, U., Vinjé, J. et al. (2014). Infection control for norovirus. Clinical Microbiology and Infection. 20, pp. 731-740. doi: 10.1111/1469-0691.12674.

Bartsch, S.M., Lopman, B.A., Ozawa, S., Hall, A.J. and Lee, B.Y. (2016). Global Economic Burden of Norovirus Gastroenteritis. PLOS ONE. 11, pp. e0151219.

Benabbes, L., Ollivier, J., Schaeffer, J., Parnaudeau, S., Rhaissi, H., Nourlil, J. et al. (2013). Norovirus and Other Human Enteric Viruses in Moroccan Shellfish. Food and Environmental Virology. 5, pp. 35-40. doi: 10.1007/s12560-012-9095-8.

Bernstein, D.I., Atmar, R.L., Lyon, G.M., Treanor, J.J., Chen, W.H., Jiang, X. et al. (2015). Norovirus Vaccine Against Experimental Human GII.4 Virus Illness: A Challenge Study in Healthy Adults. Journal of Infectious Diseases. 211, pp. 870-878. doi: 10.1093/infdis/jiu497.

Bitler, E.J., Matthews, J.E., Dickey, B.W., Eisenberg, J.N.S. and Leon, J.S. (2013). Norovirus outbreaks: a systematic review of commonly implicated transmission routes and vehicles. Epidemiology and infection. 141, pp. 1563-1571. doi: 10.1017/S095026881300006X.

Borchardt, M.A., Spencer, S.K., Kieke, B.A., Lambertini, E. and Loge, F.J. (2012). Viruses in nondisinfected drinking water from municipal wells and community incidence of acute gastrointestinal illness. Environmental Health Perspectives. 120, pp. 1272-9.

Borchardt, M.A., Spencer, S.K., Kieke, B.A., Lambertini, E. and Loge, F.J. (2012). Viruses in Nondisinfected Drinking Water from Municipal Wells and Community Incidence of Acute Gastrointestinal Illness. Environmental Health Perspectives. 120, pp. 1272-1279. doi: 10.1289/ehp.1104499.

Campos, C.J.A., Kershaw, S., Morgan, O.C. and Lees, D.N. (2017). Risk factors for norovirus contamination of shellfish water catchments in England and Wales. International Journal of Food Microbiology. 241, pp. 318-324. doi: 10.1016/j.ijfoodmicro.2016.10.028.

Cannon, J.L., Papafragkou, E., Park, G.W., Osborne, J., Jaykus, L.A. and Vinjé, J. (2006). Surrogates for the study of norovirus stability and inactivation in the environment: A comparison of murine norovirus and feline calicivirus. Journal of Food Protection. 69, pp. 2761-2765.

Carducci, A. and Verani, M. (2013). Effects of bacterial, chemical, physical and meteorological variables on virus removal 
by a wastewater treatment plant. Food and Environmental Virology. 5, pp. 69-76. doi: 10.1007/s12560-013-9105-5.

Hall, A.J., Vinjé, J., Lopman, B., Park, G.W., Yen, C., Gregoricus, N. et al. (2011). Updated Norovirus Outbreak Management and Disease Prevention Guidelines. MMWR Recommendations and Reports. 60, pp. 1-18.

Chan, M.C.W., Sung, J.J.Y., Lam, R.K.Y., Chan, P.K.S., Lee, N.L.S., Lai, R.W.M. et al. (2006). Fecal viral load and norovirusassociated gastroenteritis. Emerging Infectious Diseases. 12, pp. 1278-1280. doi: 10.3201/eid1208.060081.

Cheesbrough, J.S., Barkess-Jones, L. and Brown, D.W. (1997). Possible prolonged environmental survival of small round structured viruses [4]. Journal of Hospital Infection. 35, pp. 325-326. doi: 10.1016/S0195-6701(97)90230-9.

Czakó, R., Atmar, R.L., Opekun, A.R., Gilger, M.A., Graham, D.Y. and Estes, M.K. (2015). Experimental human infection with norwalk virus elicits a surrogate neutralizing antibody response with cross-genogroup activity. Clinical and Vaccine Immunology. 22, pp. 221-8. doi: 10.1128/CVI.00516-14.

Dancer, D., Rangdale, R.E., Lowther, J.a and Lees, D.N. (2010). Human norovirus RNA persists in seawater under simulated winter conditions but does not bioaccumulate efficiently in Pacific Oysters (Crassostrea gigas). Journal of Food Protection. 73, pp. 2123-2127.

Da Silva, A.K., Le Guyader, F.S., Le Saux, J.C., Pommepuy, M., M ontgomery, M.A. and Elimelech, M. (2008)Norovirus removal and particle association in a waste stabilization pond. Environmental Science and Technology. 42, pp. 9151-7.

Da Silva, A.K., Le Saux, J.C., Parnaudeau, S., Pommepuy, M., Elimelech, M. and Le Guyader, F.S. (2007). Evaluation of removal of noroviruses during wastewater treatment, using real-time reverse transcription-PCR: Different behaviors of genogroups I and II. Applied and Environmental Microbiology. 73, pp. 7891-7897. doi: 10.1128/AEM.01428-07.

de la Noue, A.C., Estienney, M., Aho, S., Perrier-Cornet, J.M., de Rougemont, A., Pothier, P. et al. (2014). Absolute humidity influences the seasonal persistence and infectivity of human norovirus. Applied and Environmental Microbiology. 80, pp. 7196-7205. doi: 10.1128/AEM.01871-14.

El-Senousy, W.M., Costafreda, I.M., Pinto, R.M. and Bosch, A. (2013). Method validation for norovirus detection in naturally contaminated irrigation water and fresh produce. International Journal of Food Microbiology. 167, pp. 74-79. doi: 10.1016/j.ijfoodmicro.2013.06.023.

Escudero, B.I., Rawsthorne, H., Gensel, C. and Jaykus, L.a. (2012). Persistence and Transferability of Noroviruses on and between Common Surfaces and Foods. Journal of Food Protection. 75, pp. 927-935. doi: 10.4315/0362-028X.JFP-11-460.

Flannery, J., Rajko-Nenow, P., Keaveney, S., O'Flaherty, V. and Dore, W. (2013). Simulated sunlight inactivation of norovirus and FRNA bacteriophage in seawater. Journal of Applied Microbiology. 115, pp. 915-922. doi: 10.1111/jam.12279.

Gentry, J., Vinjé, J., Guadagnoli, D. and Lipp, E.K. (2009). Norovirus distribution within an estuarine environment. Applied and Environmental Microbiology. 75, pp. 5474-5480. doi: 10.1128/AEM.00111-09.

Hall, A.J., Lopman, B.A., Payne, D.C., Patel, M.M., Gastañaduy, P.A., Vinjé, J. et al. (2013). Norovirus disease in the united states. Emerging Infectious Diseases. 19, pp. 1198-1205. doi: 10.3201/eid1908.130465. 
Hall, A.J., Vinjé, J., Lopman, B., Park, G.W., Yen, C., Gregoricus, N. et al. (2011). Updated Norovirus Outbreak Management and Disease Prevention Guidelines. MMWR Recommendations and Reports. 60, pp. 1-15.

Hamza, I.A., Jurzik, L., Stang, A., Sure, K., Uberla, K. and Wilhelm, M. (2009). Detection of human viruses in rivers of a densly-populated area in Germany using a virus adsorption elution method optimized for PCR analyses. Water Research. 43, pp. 2657-2668. doi: 10.1016/j.watres.2009.03.020.

Haramoto, E., Katayama, H., Oguma, K., Yamashita, H., Tajima, A., Nakajima, H. et al. (2006). Seasonal profiles of human noroviruses and indicator bacteria in a wastewater treatment plant in Tokyo, Japan. Water Science and Technology. 54, pp. 301-308. doi: 10.2166/wst.2006.888.

Haramoto, E., Katayama, H., Phanuwan, C. and Ohgaki, S. (2008). Quantitative detection of sapoviruses in wastewater and river water in Japan. Letters in Applied Microbiology. 46, pp. 408-413. doi: 10.1111/j.1472-765X.2008.02330.x.

Haramoto, E., Katayama, H., Asami, M. and Akiba, M. (2012). Development of a novel method for simultaneous concentration of viruses and protozoa from a single water sample. Journal of Virological Methods. 182, pp. 62-69. doi: 10.1016/j.jviromet.2012.03.011.

Haramoto, E., Katayama, H., Oguma, K. and Ohgaki, S. (2005). Application of cation-coated filter method to detection of noroviruses, enteroviruses, adenoviruses, and torque teno viruses in the Tamagawa River in Japan. Applied and Environmental Microbiology. 71, pp. 2403-11. doi: 10.1128/AEM.71.5.2403-2411.2005.

Haramoto, E., Kitajima, M., Kishida, N., Katayama, H., Asami, M. and Akiba, M. (2012). Occurrence of Viruses and Protozoa in Drinking Water Sources of Japan and Their Relationship to Indicator Microorganisms. Food and Environmental Virology. 4, pp. 93-101. doi: 10.1007/s12560-012-9082-0.

Harding, A.S. and Schwab, K.J. (2012). Using limes and synthetic psoralens to enhance solar disinfection of water (SODIS): A laboratory evaluation with norovirus, Escherichia coli, and MS2. American Journal of Tropical Medicine and Hygiene. 86, pp. 566-572. doi: 10.4269/ajtmh.2012.11-0370.

Hata, A., Katayama, H., Kojima, K., Sano, S., Kasuga, I., Kitajima, M. et al. (2014). Effects of rainfall events on the occurrence and detection efficiency of viruses in river water impacted by combined sewer overflows. The Science of the Total Environment. 468-469, pp. 757-63.

Hata, A., Katayama, H., Kitajima, M., Visvanathan, C., Nol, C. and Furumai, H. (2011). Validation of internal controls for extraction and amplification of nucleic acids from enteric viruses in water samples. Applied and Environmental Microbiology. 77, pp. 4336-4343. doi: 10.1128/AEM.00077-11.

Hennechart-Collette, C., Martin-Latil, S., Guillier, L. and Perelle, S. (2015). Determination of which virus to use as a process control when testing for the presence of hepatitis A virus and norovirus in food and water. International Journal of Food Microbiology. 202, pp. 57-65. doi: 10.1016/j.ijfoodmicro.2015.02.029.

Herbst-Kralovetz, M., Mason, H.S. and Chen, Q. (2010). Norwalk virus-like particles as vaccines. Expert Review of Vaccines. 9, pp. 299-307. doi: 10.1586/erv.09.163. 
Hirneisen, K.A. and Kniel, K.E. (2013). Norovirus surrogate survival on spinach during preharvest growth. Phytopathology. 103, pp. 389-94. doi: 10.1094/PHYTO-09-12-0231-FI.

ISO (2013). Microbiology of food and animal feed - Horizontal method for determination of hepatitis A virus and norovirus in food using real-time RT-PCR - Part 2: Method for qualitative detection. ISO/TS 15216-2.

ISO (2013). Microbiology of food and animal feed -- Horizontal method for determination of hepatitis A virus and norovirus in food using real-time RT-PCR -- Part 1: Method for quantification. ISO/TS 15216-1.

Iwai, M., Hasegawa, S., Obara, M., Nakamura, K., Horimoto, E., Takizawa, T. et al. (2009). Continuous presence of noroviruses and sapoviruses in raw sewage reflects infections among inhabitants of Toyama, Japan (2006 to 2008). Applied and Environmental Microbiology. 75, pp. 1264-1270. doi: 10.1128/AEM.01166-08.

Jin, M., He, Y., Li, H., Huang, P., Zhong, W., Yang, H. et al. (2013). Two gastroenteritis outbreaks caused by GII Noroviruses: host susceptibility and HBGA phenotypes. PloS one. 8, pp. e58605. doi: 10.1371/journal.pone.0058605.

Kageyama, T., Kojima, S., Shinohara, M., Uchida, K., Fukushi, S., Hoshino, F.B. et al. (2003). Broadly reactive and highly sensitive assay for Norwalk-like viruses based on real-time quantitative reverse transcription-PCR. Journal of Clinical Microbiology. 41, pp. 1548-1557.

Katayama, H., Haramoto, E., Oguma, K., Yamashita, H., Tajima, A., Nakajima, H. et al. (2008). One-year monthly quantitative survey of noroviruses, enteroviruses, and adenoviruses in wastewater collected from six plants in Japan. Water Research. 42, pp. 1441-8.

Keswick, B.H., Satterwhite, T.K., Johnson, P.C., DuPont, H.L., Secor, S.L., Bitsura, J.A. et al. (1985). Inactivation of Norwalk virus in drinking water by chlorine. Applied and Environmental Microbiology. 50, pp. 261-264.

Kim, A.N., Park, S.Y., Bae, S.C., Oh, M.H. and Ha, S.D. (2014). Survival of Norovirus Surrogate on Various Food-Contact Surfaces. Food and Environmental Virology. 6, pp. 182-188. doi: 10.1007/s12560-014-9154-4.

Kim, S.J., Si, J., Lee, J.E. and Ko, G. (2012). Temperature and humidity influences on inactivation kinetics of enteric viruses on surfaces. Environmental Science and Technology. 46, pp. 13303-13310. doi: 10.1021/es3032105.

Kimura, H., Nagano, K., Kimura, N., Shimizu, M., Ueno, Y., Morikane, K. et al. (2011). A norovirus outbreak associated with environmental contamination at a hotel. Epidemiology and Infection. 139, pp. 317-325. doi: 10.1017/S0950268810000981.

Kirk, M.D., Pires, S.M., Black, R.E., Caipo, M., Crump, J.A., Devleesschauwer, B. et al. (2015). World Health Organization Estimates of the Global and Regional Disease Burden of 22 Foodborne Bacterial, Protozoal, and Viral Diseases, 2010: A Data Synthesis. PLOS Medicine. 12, Public Library of Science. pp. e1001921.

Kitajima, M., Iker, B.C., Pepper, I.L. and Gerba, C.P. (2014). Relative abundance and treatment reduction of viruses during wastewater treatment processes-identification of potential viral indicators. The Science of the Total Environment. 488, pp. 290-296.

Kitajima, M., Haramoto, E., Phanuwan, C., Katayama, H. and Furumai, H. (2012). Molecular detection and genotyping of human noroviruses in influent and effluent water at a wastewater treatment plant in Japan. Journal of Applied 
Microbiology. 112, pp. 605-613. doi: 10.1111/j.1365-2672.2012.05231.x.

Kitajima, M., Haramoto, E., Phanuwan, C., Katayama, H. and Ohgaki, S. (2009). Detection of genogroup IV norovirus in wastewater and river water in Japan. Letters in Applied Microbiology. 49, pp. 655-658. doi: $10.1111 / \mathrm{j} .1472-765 \mathrm{X} .2009 .02718 . x$.

Kitajima, M., Haramoto, E., Phanuwan, C. and Katayama, H. (2011). Genotype distribution of human sapoviruses in wastewater in Japan. Applied and Environmental Microbiology. 77, pp. 4226-4229. doi: 10.1128/AEM.00088-11.

Kitajima, M., Oka, T., Haramoto, E., Katayama, H., Takeda, N., Katayama, K. et al. (2010). Detection and genetic analysis of human sapoviruses in river water in Japan. Applied and Environmental Microbiology. 76, pp. 2461-2467. doi: 10.1128/AEM.02739-09.

Kitajima, M., Oka, T., Haramoto, E., Takeda, N., Katayama, K. and Katayama, H. (2010). Seasonal distribution and genetic diversity of genogroups I, II, and IV noroviruses in the Tamagawa River, Japan. Environmental Science and Technology. 44, pp. 7116-7122. doi: 10.1021/es100346a.

Kiulia, N.M., Netshikweta, R., Page, N.A., Van Zyl, W.B., Kiraithe, M.M., Nyachieo, A. et al. (2010). The detection of enteric viruses in selected urban and rural river water and sewage in Kenya, with special reference to rotaviruses. Journal of Applied Microbiology. 109, pp. 818-828. doi: 10.1111/j.1365-2672.2010.04710.x.

Lanata, C.F., Fischer-Walker, C.L., Olascoaga, A.C., Torres, C.X., Aryee, M.J. and Black, R.E. (2013). Global causes of diarrheal disease mortality in children $<5$ years of age: a systematic review. PLoS One. 8, pp. e72788.

La Rosa, G., Fontana, S., Di Grazia, A., Iaconelli, M., Pourshaban, M. and Muscillo, M. (2007). Molecular identification and genetic analysis of norovirus genogroups I and II in water environments: Comparative analysis of different reverse transcription-PCR assays. Applied and Environmental Microbiology. 73, pp. 4152-4161. doi: 10.1128/AEM.00222-07.

La Rosa, G., Pourshaban, M., Iaconelli, M. and Muscillo, M. (2010). Quantitative real-time PCR of enteric viruses in influent and effluent samples from wastewater treatment plants in Italy. Annali dell'Istituto Superiore di Sanita. 46, pp. 266-73. doi: Doi: 10.4415/ann_10_03_07.

La Rosa, G., Pourshaban, M., Iaconelli, M. and Muscillo, M. (2009). Quantification of Norovirus Genogroups I and II in Environmental and Clinical Samples Using TaqMan Real-Time RT-PCR. Food and Environmental Virology. 1, pp. 15-22. doi: 10.1007/s12560-008-9002-5.

Lee, B.R., Lee, S.G., Park, J.H., Kim, K.Y., Ryu, S.R., Rhee, O.J. et al. (2013). Norovirus contamination levels in ground water treatment systems used for food-catering facilities in South Korea. Viruses. 5, pp. 1646-1654. doi: $10.3390 / \mathrm{v} 5071646$.

Lee, G.C., Jheong, W.H., Jung, G.S., Oh, S.A., Kim, M.J., Rhee, O.J. et al. (2012). Detection and Molecular Characterization of Human Noroviruses in Korean Groundwater Between 2008 and 2010. Food and Environmental Virology. 4, pp. 115-123. doi: 10.1007/s12560-012-9084-y.

Lee, J.E. and Ko, G. (2013). Norovirus and MS2 inactivation kinetics of UV-A and UV-B with and without TiO2. Water Research. 47, pp. 5607-5613. doi: 10.1016/j.watres.2013.06.035. 
Lee, L.E., Cebelinski, E.A., Fuller, C., Keene, W.E., Smith, K., Vinjé, J. et al. (2012). Sapovirus Outbreaks in Long-Term Care Facilities, Oregon and Minnesota, USA, 2002-2009. Emerging Infectious Diseases. 18, Centers for Disease Control and Prevention. pp. 873-876. doi: 10.3201/eid1805.111843.

Lee, N., Chan, M.C.W., Wong, B., Choi, K.W., Sin, W., Lui, G. et al. (2007). Fecal viral concentration and diarrhea in norovirus gastroenteritis. Emerging Infectious Diseases. 13, pp. 1399-1401. doi: 10.3201/eid1309.061535.

Le Guyader, F.S., Loisy, F., Atmar, R.L., Hutson, A.M., Estes, M.K., Ruvoën-Clouet, N. et al. (2006). Norwalk virus-specific binding to oyster digestive tissues. Emerging Infectious Diseases. 12, pp. 931-936. doi: 10.3201/eid1206.051519.

Leshem, E., Wikswo, M., Barclay, L., Brandt, E., Storm, W., Salehi, E. et al. (2013). Effects and clinical significance of GII.4 Sydney norovirus, United States, 2012-2013. Emerging Infectious Diseases. 19, pp. 1231-8. doi: 10.3201/eid1908.130458.

Liang, L., Goh, S.G., Vergara, G.G., Fang, H.M., Rezaeinejad, S., Chang, S.Y. et al. (2015). Alternative fecal indicators and their empirical relationships with enteric viruses, Salmonella enterica, and Pseudomonas aeruginosa in surface waters of a tropical urban catchment. Applied and Environmental Microbiology. 81, pp. 850-860. doi: 10.1128/AEM.02670-14.

Lindesmith, L.C., Donaldson, E., Leon, J., Moe, C.L., Frelinger, J.A., Johnston, R.E. et al. (2010). Heterotypic humoral and cellular immune responses following Norwalk virus infection. Journal of Virology. 84, pp. 1800-1815. doi: 10.1128/JVI.02179-09.

Lindesmith, L.C., Ferris, M.T., Mullan, C.W., Ferreira, J., Debbink, K., Swanstrom, J. et al. (2015). Broad Blockade Antibody Responses in Human Volunteers after Immunization with a Multivalent Norovirus VLP Candidate Vaccine: Immunological Analyses from a Phase I Clinical Trial. PLOS Medicine. 12, pp. e1001807. doi: 10.1371/journal.pmed.1001807.

Lin, Y., Fengling, L., Lianzhu, W., Yuxiu, Z. and Yanhua, J. (2014). Function of VP2 protein in the stability of the secondary structure of virus-like particles of genogroup II norovirus at different $\mathrm{pH}$ levels: Function of VP2 protein in the stability of NoV VLPs. Journal of Microbiology. 52, pp. 970-5. doi: 10.1007/s12275-014-4323-6.

Liu, P., Jaykus, L.A., Wong, E. and Moe, C. (2012). Persistence of Norwalk virus, male-specific coliphage, and Escherichia coli on stainless steel coupons and in phosphate-buffered saline. Journal of Food Protection. 75, pp. 2151-7. doi: 10.4315/0362-028X.JFP-12-197.

Lodder, W.J. and De Roda Husman, A.M. (2005). Presence of noroviruses and other enteric viruses in sewage and surface waters in The Netherlands. Applied and Environmental Microbiology. 71, pp. 1453-61. doi: 10.1128/AEM.71.3.1453-1461.2005.

Lodder, W.J. and De Roda Husman, A.M. (2005). Presence of Noroviruses and Other Enteric Viruses in Sewage and Surface Waters in The Netherlands. Applied and Environmental Microbiology. 71, pp. 1453-1461. doi: 10.1128/AEM.71.3.1453-1461.2005.

Lowther, J.A., Gustar, N.E., Powell, A.L., Hartnell, R.E. and Lees, D.N. (2012). Two-year systematic study to assess norovirus contamination in oysters from commercial harvesting areas in the United Kingdom. Applied and Environmental Microbiology. 78, pp. 5812-5817. doi: 10.1128/AEM.01046-12.

MacCannell, T., Umscheid, C.A., Agarwal, R.K., Lee, I., Kuntz, G. and Stevenson, K.B. (2011). Guideline for the prevention and control of norovirus gastroenteritis outbreaks in healthcare settings. Infection Control and Hospital Epidemiology. 32, 
pp. 939-969. doi: 10.1086/662025.

Macinga, D.R., Sattar, S.A., Jaykus, L.A. and Arbogast, J.W. (2008). Improved inactivation of nonenveloped enteric viruses and their surrogates by a novel alcohol-based hand sanitizer. Applied and Environmental Microbiology. 74, pp. 5047-5052. doi: 10.1128/AEM.00487-08.

Makaya, J.M., Kaplon, J., Fremy, C., Barro, N., Aho, S., Pothier, P. et al. (2015). Norovirus and rotavirus survival in urine collected from a public ecological sanitation system in Ouagadougou, Burkina Faso. Food and Environmental Virology. 7, pp. 41-48. doi: 10.1007/s12560-014-9172-2.

Marionneau, S., Ruvoën, N., Le Moullac-Vaidye, B., Clement, M., Cailleau-Thomas, A., Ruiz-Palacois, G. et al. (2002). Norwalk virus binds to histo-blood group antigens present on gastroduodenal epithelial cells of secretor individuals. Gastroenterology. 122, pp. 1967-1977. doi: 10.1053/gast.2002.33661.

Masclaux, F.G., Hotz, P., Friedli, D., Savova-Bianchi, D. and Oppliger, A. (2013). High occurrence of hepatitis E virus in samples from wastewater treatment plants in Switzerland and comparison with other enteric viruses. Water Research. 47, pp. 5101-5109. doi: 10.1016/j.watres.2013.05.050.

Matsushita, T., Shirasaki, N., Tatsuki, Y. and Matsui, Y. (2013). Investigating norovirus removal by microfiltration, ultrafiltration, and precoagulation-microfiltration processes using recombinant norovirus virus-like particles and real-time immuno-PCR. Water Research. 47, pp. 5819-5827. doi: 10.1016/j.watres.2013.07.004.

Mayo, M.A. (2002). A summary of taxonomic changes recently approved by ICTV. Archives of Virology. 8, pp. 1-2.

Miura, T., Okabe, S., Nakahara, Y. and Sano, D. (2015). Removal properties of human enteric viruses in a pilot-scale membrane bioreactor (MBR) process. Water Research. 75, pp. 282-291. doi: 10.1016/j.watres.2015.02.046.

Nordgren, J., Matussek, A., Mattsson, A., Svensson, L. and Lindgren, P.E. (2009). Prevalence of norovirus and factors influencing virus concentrations during one year in a full-scale wastewater treatment plant. Water Research. 43, pp. 1117-1125. doi: 10.1016/j.watres.2008.11.053.

Nordgren, J., Matussek, A., Mattsson, A., Svensson, L. and Lindgren, P.E. (2009). Prevalence of norovirus and factors influencing virus concentrations during one year in a full-scale wastewater treatment plant. Water Research. 43, pp. 1117-1125. doi: 10.1016/j.watres.2008.11.053.

Oka, T., Wang, Q., Katayama, K. and Saif, L.J. (2015). Comprehensive Review of Human Sapoviruses. Clinical Microbiology Reviews. 28, pp. 32-53. doi: 10.1128/CMR.00011-14.

Ottoson, J., Hansen, A., Bjorlenius, B., Norder, H. and Stenström, T.A. (2006). Removal of viruses, parasitic protozoa and microbial indicators in conventional and membrane processes in a wastewater pilot plant. Water Research. 40, pp. 1449-1457. doi: 10.1016/j.watres.2006.01.039.

Pérez-Sautu, U., Sano, D., Guix, S., Kasimir, G., Pinto, R.M. and Bosch, A. (2012). Human norovirus occurrence and diversity in the Llobregat river catchment, Spain. Environmental Microbiology. 14, pp. 494-502. doi: 10.1111/j.1462-2920.2011.02642.x. 
Park, G.W., Barclay, L., Macinga, D., Charbonneau, D., Pettigrew, C.A. and Vinjé, J. (2010). Comparative efficacy of seven hand sanitizers against murine norovirus, feline calicivirus, and GII.4 norovirus. Journal of Food Protection. 73, pp. 2232-2238.

Park, S.Y. and Ha, S.D. (2015). Inactivation of murine norovirus-1 and hepatitis A virus in the Korean traditional preserved raw crab product Ganjanggejang by soy sauce during storage. Food Control. 51, pp. 293-299. doi: 10.1016/j.foodcont.2014.11.046.

Payne, D.C., Vinjé, J., Szilagyi, P.G., Edwards, K.M., Staat, M.A., Weinberg, G.A. et al. (2013). Norovirus and medically attended gastroenteritis in U.S. children. The New England Journal of Medicine. 368, pp. 1121-1130. doi: 10.1056/NEJMsa1206589.

Phillips, G. (2011). Risk factors for symptomatic and asymptomatic norovirus infection in the community. Epidemiology and Infection. 139, pp. 1-11. doi: 10.1017/S0950268810002839.

Phillips, G., Tam, C.C., Conti, S., Rodrigues, L.C., Brown, D., Iturriza-Gómara, M. et al. (2010). Community incidence of norovirus-associated infectious intestinal disease in England: Improved estimates using viral load for norovirus diagnosis. American Journal of Epidemiology. 171, pp. 1014-1022. doi: 10.1093/aje/kwq021.

Pires, S.M., Fischer-Walker, C.L., Lanata, C.F., Devleesschauwer, B., Hall, A.J., Kirk, M.D. et al. (2015). Aetiology-Specific Estimates of the Global and Regional Incidence and Mortality of Diarrhoeal Diseases Commonly Transmitted through Food. PLoS One. 10, (Selvey, L.A., ed.). pp. e0142927. doi: 10.1371/journal.pone.0142927.

Prasad, B.V., Hardy, M.E., Dokland, T., Bella, J., Rossmann, M.G. and Estes, M.K. (1999). X-ray crystallographic structure of the Norwalk virus capsid. Science (New York, N.Y.). 286, pp. 287-290. doi: 10.1126/science.286.5438.287.

Rönnqvist, M., Mikkelä, A., Tuominen, P., Salo, S. and Maunula, L. (2014). Ultraviolet Light Inactivation of Murine Norovirus and Human Norovirus GII: PCR May Overestimate the Persistence of Noroviruses Even When Combined with Pre-PCR Treatments. Food and Environmental Virology. 6, pp. 48-57. doi: 10.1007/s12560-013-9128-y.

Rezaeinejad, S., Vergara, G.G., Woo, C.H., Lim, T.T., Sobsey, M.D. and Gin, K.Y.H. (2014). Surveillance of enteric viruses and coliphages in a tropical urban catchment. Water Research. 58, pp. 122-131. doi: 10.1016/j.watres.2014.03.051.

Saiki, R.K., Scharf, S., Faloona, F., Mullis, K.B., Horn, G.T., Erlich, H.A. et al. (1985). Enzymatic amplification of betaglobin genomic sequences and restriction site analysis for diagnosis of sickle cell anemia. Science. 80, pp. 1350 LP-1354.

Sano, D., Amarasiri, M., Hata, A., Watanabe, T. and Katayama, H. (2016). Risk management of viral infectious diseases in wastewater reclamation and reuse: Review. Environment International. 91, pp. 220-229. doi: 10.1016/j.envint.2016.03.001.

Sano, D., Pérez-Sautu, U., Guix, S., Pintó, R.M., Miura, T., Okabe, S. et al. (2011). Quantification and genotyping of human sapoviruses in the Llobregat river catchment, Spain. Applied and Environmental Microbiology. 77, pp. 1111-1114. doi: 10.1128/AEM.01721-10.

Schaeffer, J., Le Saux, J.C., Lora, M., Atmar, R.L. and Le Guyader, F.S. (2013). Norovirus contamination on French marketed oysters. International Journal of Food Microbiology. 166, pp. 244-248. doi: 10.1016/j.ijfoodmicro.2013.07.022. 
Seitz, S.R., Leon, J.S., Schwab, K.J., Lyon, M.G., Dowd, M., McDaniels, M. et al. (2011). Norovirus infectivity in humans and persistence in water. Applied and Environmental Microbiology. 77, pp. 6884-6888. doi: 10.1128/AEM.05806-11.

Shin, G.A. and Sobsey, M.D. (2015). Removal of norovirus from water by coagulation, flocculation and sedimentation processes. Water Science and Technology: Water Supply. 15, pp. 158. doi: 10.2166/ws.2014.100.

Shin, G.A. and Sobsey, M.D. (2008). Inactivation of norovirus by chlorine disinfection of water. Water Research. 42, pp. 4562-4568. doi: 10.1016/j.watres.2008.08.001.

Shin, G.A. and Sobsey, M.D. (2003). Reduction of Norwalk virus, poliovirus 1, and bacteriophage MS2 by ozone disinfection of water. Applied and Environmental Microbiology. 69, pp. 3975-3978. doi: 10.1128/AEM.69.7.3975-3978.2003.

Sickbert-Bennett, E.E., Weber, D.J., Gergen-Teague, M.F., Sobsey, M.D., Samsa, G.P. and Rutala, W.A. (2005). Comparative efficacy of hand hygiene agents in the reduction of bacteria and viruses. American Journal of Infection Control. 33, pp. 67-77. doi: 10.1016/j.ajic.2004.08.005.

Siebenga, J.J., Vennema, H., Zheng, D.P., Vinjé, J., Lee, B.E., Pang, X.L. et al. (2009). Norovirus Illness Is a Global Problem: Emergence and Spread of Norovirus GII.4 Variants, 2001-2007. The Journal of Infectious Diseases. 200, pp. 802-812. doi: $10.1086 / 605127$.

Silverman, A.I., Akrong, M.O., Amoah, P., Drechsel, P. and Nelson, K.L. (2013). Quantification of human norovirus GII, human adenovirus, and fecal indicator organisms in wastewater used for irrigation in Accra, Ghana. Journal of Water and Health. 11, pp. 473-88. doi: 10.2166/wh.2013.025.

Sima, L.C., Schaeffer, J., Le Saux, J.C., Parnaudeau, S., Elimelech, M. and Le Guyader, F.S. (2011). Calicivirus removal in a membrane bioreactor wastewater treatment plant. Applied and Environmental Microbiology. 77, pp. 5170-5177. doi: 10.1128/AEM.00583-11.

Simmons, F.J., Kuo, D.H.W. and Xagoraraki, I. (2011). Removal of human enteric viruses by a full-scale membrane bioreactor during municipal wastewater processing. Water Research. 45, pp. 2739-2750. doi: 10.1016/j.watres.2011.02.001.

Skraber, S., Ogorzaly, L., Helmi, K., Maul, A., Hoffmann, L., Cauchie, H.M. et al. (2009). Occurrence and persistence of enteroviruses, noroviruses and F-specific RNA phages in natural wastewater biofilms. Water Research. 43, pp. 4780-4789. doi: 10.1016/j.watres.2009.05.020.

Stals, A., Mathijs, E., Baert, L., Botteldoorn, N., Denayer, S., Mauroy, A. et al. (2012). Molecular Detection and Genotyping of Noroviruses. Food and Environmental Virology. 4, pp. 153-167. doi: 10.1007/s12560-012-9092-y.

Tacket, C.O., Sztein, M.B., Losonsky, G.A., Wasserman, S.S. and Estes, M.K. (2003). Humoral, mucosal, and cellular immune responses to oral Norwalk virus-like particles in volunteers. Clinical Immunology. 108, pp. 241-247. doi: 10.1016/S1521-6616(03)00120-7.

Teunis, P.F.M., Moe, C.L., Liu, P., Miller, S.E., Lindesmith, L., Baric, R.S. et al. (2008). Norwalk virus: how infectious is it?. Journal of Medical Virology. 80, pp. 1468-1476. doi: 10.1002/jmv.21237. 
Ueki, Y., Sano, D., Watanabe, T., Akiyama, K. and Omura, T. (2005). Norovirus pathway in water environment estimated by genetic analysis of strains from patients of gastroenteritis, sewage, treated wastewater, river water and oysters. Water Research. 39, pp. 4271-4280. doi: 10.1016/j.watres.2005.06.035.

Uhrbrand, K., Schultz, A.C. and Madsen, A.M. (2011). Exposure to Airborne Noroviruses and Other Bioaerosol Components at a Wastewater Treatment Plant in Denmark. Food and Environmental Virology. 3, pp. 130-137. doi: 10.1007/s12560-011-9068-3.

Verhaelen, K., Bouwknegt, M., Lodder-Verschoor, F., Rutjes, S.A. and De Roda Husman, A.M. (2012). Persistence of human norovirus GII.4 and GI.4, murine norovirus, and human adenovirus on soft berries as compared with PBS at commonly applied storage conditions. International Journal of Food Microbiology. 160, pp. 137-144. doi: 10.1016/j.jjfoodmicro.2012.10.008.

Victoria, M., Rigotto, C., Moresco, V., de Abreu Correa, A., Kolesnikovas, C., Leite, J.P.G. et al. (2010). Assessment of norovirus contamination in environmental samples from Florianópolis City, Southern Brazil. Journal of Applied Microbiology. 109, pp. 231-238. doi: 10.1111/j.1365-2672.2009.04646.x.

Vinjé, J. (2015). Advances in Laboratory Methods for Detection and Typing of Norovirus. Journal of Clinical Microbiology. 53, pp. 373-381. doi: 10.1128/JCM.01535-14.

Wei, J., Jin, Y., Sims, J.T. and Kniel, K.E. (2010). Fate of human enteric viruses during dairy manure-based composting. Journal of Food Protection. 73, pp. 1543-7.

Wolf, S., Hewitt, J. and Greening, G.E. (2010). Viral multiplex quantitative PCR assays for tracking sources of fecal contamination. Applied and Environmental Microbiology. 76, pp. 1388-94. doi: 10.1128/AEM.02249-09.

Yang, N., Qi, H., Wong, M.M.L., Wu, R.S.S. and Kong, R.Y.C. (2012). Prevalence and diversity of norovirus genogroups I and II in Hong Kong marine waters and detection by real-time PCR. Marine Pollution Bulletin. 64, pp. 164-168. doi: 10.1016/j.marpolbul.2011.10.037. 